\title{
Knowledge Domain and Emerging Trends in Ferroptosis Research: A Bibliometric and Knowledge-Map Analysis
}

OPEN ACCESS

Edited by:

Maryam Mehrpour.

INSERM U1151 Institut Necker

enfants Malades, France

Reviewed by:

Andy Wai Kan Yeung,

The University of Hong Kong, China

Carlos Mulet Forteza,

University of the Balearic Islands,

Spain

*Correspondence:

Jianqing Ju

jujianqing@163.com

Hao Xu

xuhaotcm@hotmail.com

Specialty section:

This article was submitted to Molecular and Cellular Oncology,

a section of the journal

Frontiers in Oncology

Received: 27 March 2021

Accepted: 05 May 2021

Published: 03 June 2021

Citation:

Zhang J, Song L, XU L, Fan Y, Wang T, Tian W, Ju J and Xu H (2021) Knowledge Domain and Emerging Trends in Ferroptosis

Research: A Bibliometric and Knowledge-Map Analysis.

Front. Oncol. 11:686726.

doi: 10.3389/fonc.2021.686726

\begin{abstract}
Jie Zhang ${ }^{1,2}$, Luxia Song ${ }^{1,2}$, Liyan $X u^{3}$, Yixuan Fan ${ }^{1,2}$, Tong Wang ${ }^{1,2}$, Wende Tian ${ }^{2,4}$, Jianqing $\mathrm{Ju}^{2 *}$ and $\mathrm{HaO} \mathrm{Xu}^{2 *}$

${ }^{1}$ Graduate School, Beijing University of Chinese Medicine, Beijing, China, ${ }^{2}$ National Clinical Research Center for Chinese Medicine Cardiology, Xiyuan Hospital, China Academy of Chinese Medical Sciences, Beijing, China, ${ }^{3}$ Department of Computer Science, Emory University, Atlanta, GA, United States, ${ }^{4}$ Graduate School, China Academy of Chinese Medical Sciences, Beijing, China
\end{abstract}

Objectives: To identify the cooperation and impact of authors, countries, institutions, and journals, evaluate the knowledge base, find the hotspot trends, and detect the emerging topics regarding ferroptosis research.

Methods: The articles and reviews related to ferroptosis were obtained from the Web of Science Core Collection on November 1, 2020. Two scientometric software (CiteSpace 5.7 and VOSviewer 1.6.15) were used to perform bibliometric and knowledge-map analysis.

Results: A total of 1,267 papers were included, in 466 academic journals by 6,867 authors in 438 institutions from 61 countries/regions. The ferroptosis-related publications were increasing rapidly. Cell Death \& Disease published the most papers on ferroptosis, while Cell was the top co-cited journal, publication journals and co-cited journals were major in the molecular and biology fields. The United States and China were the most productive countries; meanwhile, the University of Pittsburgh, Columbia University and Guangzhou Medical University were the most active institutions. Brent R Stockwell published the most papers, while Scott J Dixon had the most co-citations; simultaneously, active cooperation existed in ferroptosis researchers. Ten references on reviews, mechanisms, and diseases were regarded as the knowledge base. Five main aspects of ferroptosis research included regulation mechanisms, nervous system injury, cancer, relationships with other types of cell death, and lipid peroxidation. The latest hotspots were nanoparticle, cancer therapy, iron metabolism, and in-depth mechanism. Notably Nrf2 might have turning significance. The emerging topics on ferroptosis research were the further molecular mechanism of ferroptosis and the wider application of ferroptosis-related disease with advanced technology. 
Conclusion: This study performed a full overview of the ferroptosis research using bibliometric and visual methods. The information would provide helpful references for scholars focusing on ferroptosis.

Keywords: ferroptosis, bibliometric, knowledge-map, CiteSpace, VOSviewer

\section{INTRODUCTION}

Ferroptosis is an iron-dependent regulated necrosis caused by unrestricted lipid peroxidation and subsequent membrane damage (1). Scott J Dixon (2) proposed the term ferroptosis in 2012 to describe a type of cell death induced by the small molecule erastin, which could lead to glutathione (GSH) depletion and glutathione peroxidase 4 (GPX4) inactivation $(1,3)$. In addition to the necrotic morphological changes, ferroptotic cell death usually shows mitochondrial abnormalities, such as increased membrane density, reduced or absent crista, condensation or swelling, and rupture of the outer membrane $(2,4-7)$. Existing evidence proves that ferroptosis plays an important role in the development of many diseases $(7,8)$, such as cancer, neurodegenerative diseases, ischemia/reperfusion injury, acute kidney injury, atherosclerosis, chronic obstructive pulmonary disease, and immune system diseases. Therefore, as an evolutionary program offers various druggable nodes, ferroptosis is supposed to be an emerging way to cure many kinds of diseases (9-14). Especially as a therapeutic model in cancer treatment and prevention of ischemic organ damage, ferroptosis has been convincingly established (15).

According to its great potential, ferroptosis has gained scholars' keen interest in better understanding the process of ferroptosis with a rapidly increasing number of publications (16). Many scholars have reviewed ferroptosis research from various aspects. For instance, Daolin Tang etc. (7) summarized the progress of ferroptosis research mainly from molecular mechanisms, including hallmarks, regulation, oxidant system, antioxidant system, membrane repair, degradation systems, transcription factors and cofactors, epigenetic regulation, assays, and the implications in disease. Xuejun Jiang etc. (17) overviewed ferroptosis from the mechanisms, pathways, biological functions in tumor suppression and immune surveillance, and the implications in cancer and ischemic damage. Marcus Conrad etc. (12) outlined ferroptosis mainly from its effects on as-yet- incurable disease, including ischemiareperfusion injury, organ failure, neurodegenerative disease and therapy-resistant cancer. However, there is no comprehensive and objective report on the publication trends, influential authors or institutions and their cooperation, knowledge base, hotspots evolution, or the emerging topics in ferroptosis research to our knowledge.

Nowadays, there are kinds of approaches to systemically review a research field, of which bibliometrics is one of the most popular methods (18). Bibliometrics can not only qualitatively and quantitatively analyze the contribution and cooperation of authors, institutions, countries, and journals, but also evaluate the development and emerging trends in scientific research (18-23). That which other methods, such as traditional review, meta-analysis, or experiment research, cannot perform. According to the strengths, it is becoming increasingly important in evaluating research trends and formulating guidelines (24). Therefore, bibliometrics is suitable for evaluating and overviewing ferroptosis research.

This study aimed to use two commonly used bibliometric tools, CiteSpace and VOSviewer, to objectively describe the knowledge domain and emerging trends of ferroptosis research from three aspects as follows. (1) We designed to quantify and identify the general information in ferroptosis research, such as the individual impact and the cooperation information, by analyzing annual publications, journals, co-cited journals, countries/regions, institutions, authors and co-cited authors. (2) We planned to find and analyze the most co-cited papers by co-cited reference analysis to evaluate the knowledge base of ferroptosis. (3) Most importantly, finding the knowledge structure and hotspots evolution, and detecting the emerging topics of ferroptosis by keywords analysis and co-cited reference burst analysis. Overall, these three aspects cover the status quo and trends of ferroptosis research.

\section{MATERIALS AND METHODS}

\section{Data Collection}

The Web of Science Core Collection (WoSCC) database is commonly used in bibliometric analysis $(19,22,25-27)$. We also chose it because it can provide comprehensive information bibliometric software needs and is regarded as the most influential database (28).

Data were retrieved from the WoSCC database on November 1, 2020. We searched "ferroptosis" and "ferroptotic" as the term and set the timespan from the inception of Web of Science (WoS) to November 1, 2020. The language was restricted to English, and the article type was limited to Article or Review. Search results were downloaded with the record content of "Full Record and Cited References" and the file format of "Plain Text". Then, we renamed the files for further analysis because CiteSpace can only recognize files named "download *.txt".

\section{Data Analysis and Visualization}

At present, the commonly used bibliometric software includes VOSViewer, CiteSpace, SCI2, NetDraw, and HistCite (29). There is no consensus on which bibliographic method is the best (30). Considering their characteristics and advantages, 
this research used both VOSviewer and CiteSpace (22, 25, 29, 31).

VOSviewer, developed by Leiden University, is a software that does well in creating, visualizing, and exploring maps based on network data $(32,33)$. We used VOSviewer 1.6 .15 to identify productive journals, co-cited journals, authors, co-cited authors, as well as the related knowledge-maps based on bibliographic data. In addition, we created the keywords co-occurrence and cluster map based on text data. Terms were obtained from titles and abstracts fields using natural language processing algorithms and complemented with a VOSviewer corpus file (33). Firstly, we cleaned the data, such as merged "van raan, a" and "vanraan, a" in author analysis, unified "glutathione" and "gsh" as "gsh," and deleted meaningless terms such as "focus" and "year" in term analysis (34). Secondly, we used fractional counting as the counting method and set the maximum number of authors per document as 25 (35). The difference between full counting and fractional counting is the strength of the links (33). The fractional counting method calculated the link strength by splitting papers according to the weight $(18,30,33)$. For example, if three authors co-author a paper, each of their link strength will be counted as $1 / 3$ in fractional counting, while it will be counted as one in full counting. It can be identified that fractional counting performs more reasonable in author analysis (36), and after comparing two methods in other sections, the data in our study showed more reasonably and clearly by the fractional counting method. Besides, in term analysis, each term was calculated a relevance score, which represented that terms with a high relevance score tend to represent specific topics, while terms with a low relevance score tend not to be representative of any specific topic (33). Therefore, we selected the terms that not only appeared more than ten times but also at the top $60 \%$ relevance score to analyze. Other thresholds (T) of items were set based on different situations (19), which were marked in corresponding tables and figures.

CiteSpace, developed by Prof. Chaomei Chen, is a bibliometric and visual analysis tool good at exploring cooperation, key points, internal structure, potential trends and dynamics in a certain field (37). Therefore, we used CiteSpace 5.7 to analyze and visualize the co-occurrence of countries/regions and institutions, dual-map of journals, trends of high-frequency keywords, cocited references, and citation bursts for references. We cleaned the data before analyzing; for instance, in countries/regions analysis, publications from Taiwan were reclassified to China (35), and those from England, Scotland, Northern Ireland, and Wales were assigned to the United Kingdom (36). Similarly, we merged the synonyms such as "GPX4" and "glutathione peroxidase 4" in keyword evolution analysis. The CiteSpace settings were as follows: time span (2012-2020), years per slice (1), pruning (Minimum Spanning Tree and Pruning Sliced Networks), selection criteria (Top $\mathrm{N}=50$ ), and others followed the default.

We used Microsoft Office Excel 2019 to manage the database and analyze the annual publications.

Besides, we obtained the 2019 impact factor (IF) and JCR division of journals from the Web of Science InCites Journal Citation Reports on November 15, 2020.

\section{RESULTS}

\section{Annual Growth Trend}

According to the data collection strategy, we collected 1,268 papers but these contained one duplicate. Finally, a total of 1,267 eligible papers were included (Annexes 1), published between 2012 and 2020. As we can see from Figure 1, ferroptosis-related references showed an annual upward tendency. Significantly, the yearly output is almost twice as much as the previous year in the last three years (2018 to present).

\section{Journals and Co-Cited Journals}

We used VOSviewer to conduct co-citation and co-cited journal analysis, finding the most active and most influential journals in the ferroptosis field. The results showed that the 1,267 references were published in 466 academic journals. Cell Death \& Disease published the most papers $(41,3.21 \%)$, followed by Biochemical and Biophysical Research Communications, Free Radical Biology and Medicine, Redox Biology, and Cell Death and Differentiation (Table 1). Among the top10 journals, seven were at the Q1 JCR division, and six had an Impact Factor (IF) of more than five (Table 1).

Among 4,781 co-cited journals, eleven journals had citations over 1,000. As we can see from Table 2, Cell had the most cocitations $(3,926,4.21 \%)$, followed by Nature, Journal of Biological Chemistry, and Proceedings of the National Academy of Sciences of the United States of America (PNAS). Among the top $10 \mathrm{co}-$ cited journals, eight were at the Q1 JCR division with an Impact Factor (IF) of more than six, seven were from the United States.

The dual-map overlay of journals stands for the topic distribution of academic journals (38) (Figure 2). The citing journals were located on the left while the cited journals were on the right, and the colored paths indicated the citation relationships. Only one primary citation path colored orange was identified, which means the studies published in Molecular/ Biology/Genetics journals were mainly cited by the studies published in Molecular/Biology/Immunology journals.

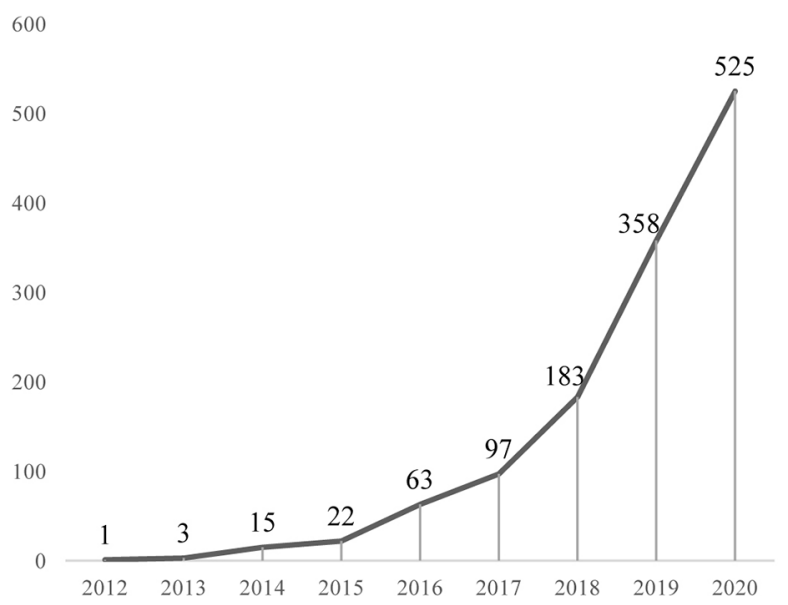

FIGURE 1 | Annual output of ferroptosis research. 
TABLE 1 | The top 10 journals of ferroptosis research.

\begin{tabular}{|c|c|c|c|c|c|}
\hline Rank & Journal & $\mathbf{N}(\%)$ & IF(2019) & JCR division & Country \\
\hline 1 & Cell Death \& Disease & $41(3.21 \%)$ & 6.304 & Q1 & UK \\
\hline 2 & Biochemical and Biophysical Research Communications & $36(2.82 \%)$ & 2.985 & Q2/Q3 & USA \\
\hline 3 & Free Radical Biology and Medicine & $31(2.43 \%)$ & 6.170 & Q1 & USA \\
\hline 4 & Redox Biology & $27(2.12 \%)$ & 9.986 & Q1 & Netherlands \\
\hline 5 & Cell Death and Differentiation & $21(1.65 \%)$ & 10.717 & Q1 & UK \\
\hline 6 & Oxidative Medicine and Cellular Longevity & $20(1.57 \%)$ & 5.076 & Q2 & USA \\
\hline 7 & International Journal of Molecular Sciences & $20(1.57 \%)$ & 4.556 & Q1/Q2 & Switzerland \\
\hline 8 & Scientific Reports & $16(1.25 \%)$ & 3.998 & Q1 & UK \\
\hline 9 & Frontiers in Neuroscience & $15(1.18 \%)$ & 3.707 & Q2 & Switzerland \\
\hline 10 & Cell Chemical Biology & $15(1.18 \%)$ & 7.739 & Q1 & USA \\
\hline
\end{tabular}

\#IF: Impact Factor.

TABLE 2 | The top 10 co-cited journals of ferroptosis research.

\begin{tabular}{|c|c|c|c|c|c|}
\hline Rank & Co-cited Journal & $\mathbf{N}(\%)$ & $\operatorname{IF}(2019)^{\#}$ & JCR division & Country \\
\hline 1 & Cell & $3,926(4.21 \%)$ & 38.637 & Q1 & USA \\
\hline 2 & Nature & $2,912(3.12 \%)$ & 42.779 & Q1 & Germany \\
\hline 3 & Journal of Biological Chemistry & $2,661(2.85 \%)$ & 4.238 & Q2 & USA \\
\hline 4 & Proceedings of The National Academy of Sciences of the United States of America & $2,475(2.65 \%)$ & 9.412 & Q1 & USA \\
\hline 5 & Cell Death and Differentiation & $1,766(1.89 \%)$ & 10.717 & Q1 & UK \\
\hline 6 & Free Radical Biology and Medicine & $1,758(1.88 \%)$ & 6.170 & Q1 & USA \\
\hline 7 & Nature Chemical Biology & $1,270(1.36 \%)$ & 12.587 & Q1 & Germany \\
\hline 8 & Plos One & $1,152(1.23 \%)$ & 2.74 & Q2 & USA \\
\hline 9 & Cancer Research & $1,066(1.14 \%)$ & 9.727 & Q1 & USA \\
\hline 10 & Science & $1,061(1.14 \%)$ & 41.846 & Q1 & USA \\
\hline
\end{tabular}

\#IF, impact factor.

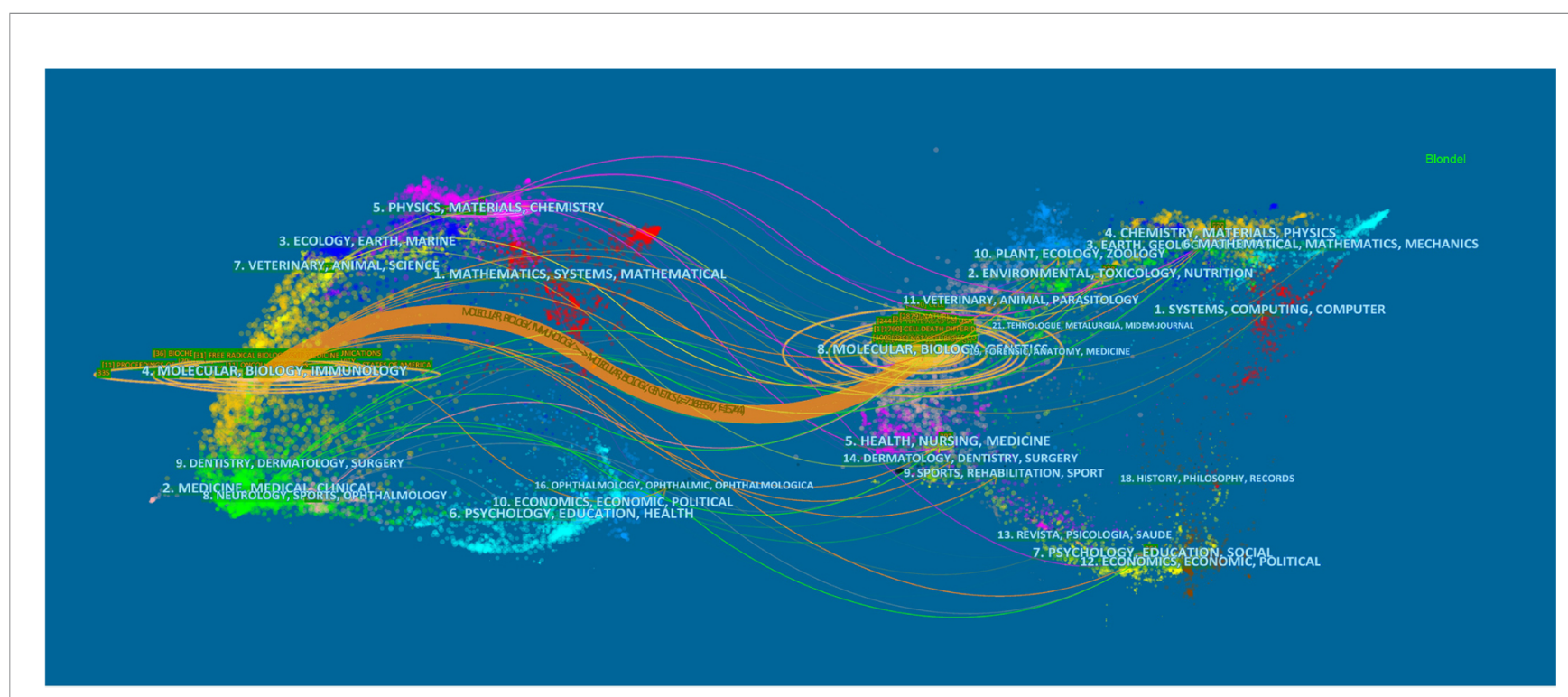

FIGURE 2 | The dual-map overlay of journals related to ferroptosis research. Notes: The citing journals were at left, the cited journals were on the right, and the colored path represents citation relationship.

\section{Countries/Regions and Institutions}

A total of 1,267 publications were co-authored by 438 institutions from 61 countries/regions. The largest number of publications were originated from China (562, 31.03\%), followed by the United States (410, 22.64\%), Germany (157, 8.67\%), and Japan (103, 5.69\%) (Table 3). Some nodes, such as the United States,
France, Germany, Australia, the United Kingdom and Canada, were colored purple round in Figure 3A in terms of their high betweenness centrality $(\geq 0.10)$, which is usually regarded as the important turning points that may lead to transformative discoveries and acts as a bridge $(37,39-41)$. Furthermore, according to the color of links, the USA (2012), Germany 
TABLE 3 | The top 10 countries/regions and institutions involved in ferroptosis research.

\begin{tabular}{|c|c|c|c|c|c|c|c|}
\hline Rank & Country/region & N (\%) & Centrality & Institution & Country/region & N (\%) & Centrality \\
\hline 1 & China & 562 (31.03\%) & 0.09 & University of Pittsburgh & USA & 59 (3.34\%) & 0.20 \\
\hline 2 & USA & $410(22.64 \%)$ & 0.18 & Columbia University & USA & 57 (3.23\%) & 0.14 \\
\hline 3 & Germany & 157 (8.67\%) & 0.31 & Guangzhou Medical University & China & $41(2.32 \%)$ & 0.08 \\
\hline 4 & Japan & 103 (5.69\%) & 0.10 & Chinese Academy of Sciences & China & $33(1.87 \%)$ & 0.08 \\
\hline 5 & France & 55 (3.04\%) & 0.39 & Zhejiang University & China & 32 (1.81\%) & 0.07 \\
\hline 6 & Australia & $49(2.71 \%)$ & 0.21 & Harvard University & USA & 29 (1.64\%) & 0.11 \\
\hline 7 & UK & $48(2.65 \%)$ & 0.19 & Stanford University & USA & $28(1.59 \%)$ & 0.10 \\
\hline 8 & Canada & $42(2.32 \%)$ & 0.15 & Central South University & China & 27 (1.53\%) & 0.03 \\
\hline 9 & Italy & $39(2.15 \%)$ & 0.01 & Jilin University & China & $25(1.42 \%)$ & 0.07 \\
\hline 10 & Russia & $33(1.82 \%)$ & 0.02 & Helmholtz Zentrum München & Germany & $25(1.42 \%)$ & 0.06 \\
\hline 10 & & & & Shanghai Jiao Tong University & China & $25(1.42 \%)$ & 0.03 \\
\hline 10 & & & & The University of Melbourne & Australia & 25 (1.42\%) & 0.10 \\
\hline
\end{tabular}

(2013), France (2013), and Russia (2013) were the earliest countries to take up the ferroptosis research. We used minimum spanning tree pruning to make the network clear (Figure 3A). Actually, no-pruning countries/regions cooccurrence map contained 61 nodes and 302 links with a density equal to 0.165 , indicating active collaborations among different countries/regions. For instance, the USA had cooperation with 34 countries/regions, followed by Germany $(\mathrm{n}=30)$, China $(\mathrm{n}=26)$, France $(\mathrm{n}=24)$, and UK $(\mathrm{n}=24)$.

The top 12 institutions were from China (6/12), USA (4/12), Germany (1/12) and Australia (1/12) (Table 3). University of Pittsburgh $(59,3.34 \%)$ published the most papers, followed by Columbia University (57, 3.23\%), Guangzhou Medical University (41, 2.32\%), Chinese Academy of Sciences (33, $1.87 \%)$, and Zhejiang University (32, 1.81\%) (Table 3).

\section{Authors and Co-Cited Authors}

A total of 6,867 authors were involved in ferroptosis research. Eighteen authors published more than ten articles. Brent R Stockwell published the most papers $(n=39)$, followed by
Daolin Tang ( $\mathrm{n}=36)$, Rui Kang ( $\mathrm{n}=35)$, Marcus Conrad $(\mathrm{n}=31)$ and Andreas Linkermann $(\mathrm{n}=26)$ (Table 4). The authors $(\mathrm{n}=135)$ who published at least five papers $(\mathrm{T} \geq 5)$ were included to build the network map of authors (Figure 4). The same color represented the same cluster. There were active collaborations in ferroptosis research, especially among authors in the same cluster, such as Brent R Stockwell and Scott J Dixon, Daolin Tang and Rui Kang, etc. Close cooperation was also observed among clusters, such as Brent R Stockwell and Andreas Linkermann, Brent R Stockwell and Marcus Conrad, Brent R Stockwell, and Xuejun Jiang, etc.

Co-cited authors are authors who have been co-cited together in a range of publications (42). Among 36,666 co-cited authors, 16 were co-cited over 200. Scott J Dixon $(n=1566)$ ranked first, followed by Wan Seok Yang ( $n=1304)$, Jose Pedro Friedmann Angeli $(n=557)$, Minghui Gao ( $\mathrm{n}=519)$, Andreas Linkermann $(\mathrm{n}=462)$, and Brent $\mathrm{R}$ Stockwell $(\mathrm{n}=462)$. The remaining four top authors were co-cited from 327 to 413 (Table 4). The authors $(n=43)$ with co-citations of at least $100(\mathrm{~T} \geq 100)$ were used to make the density map (Figure 5); this type of knowledge-map could present the high-frequency co-

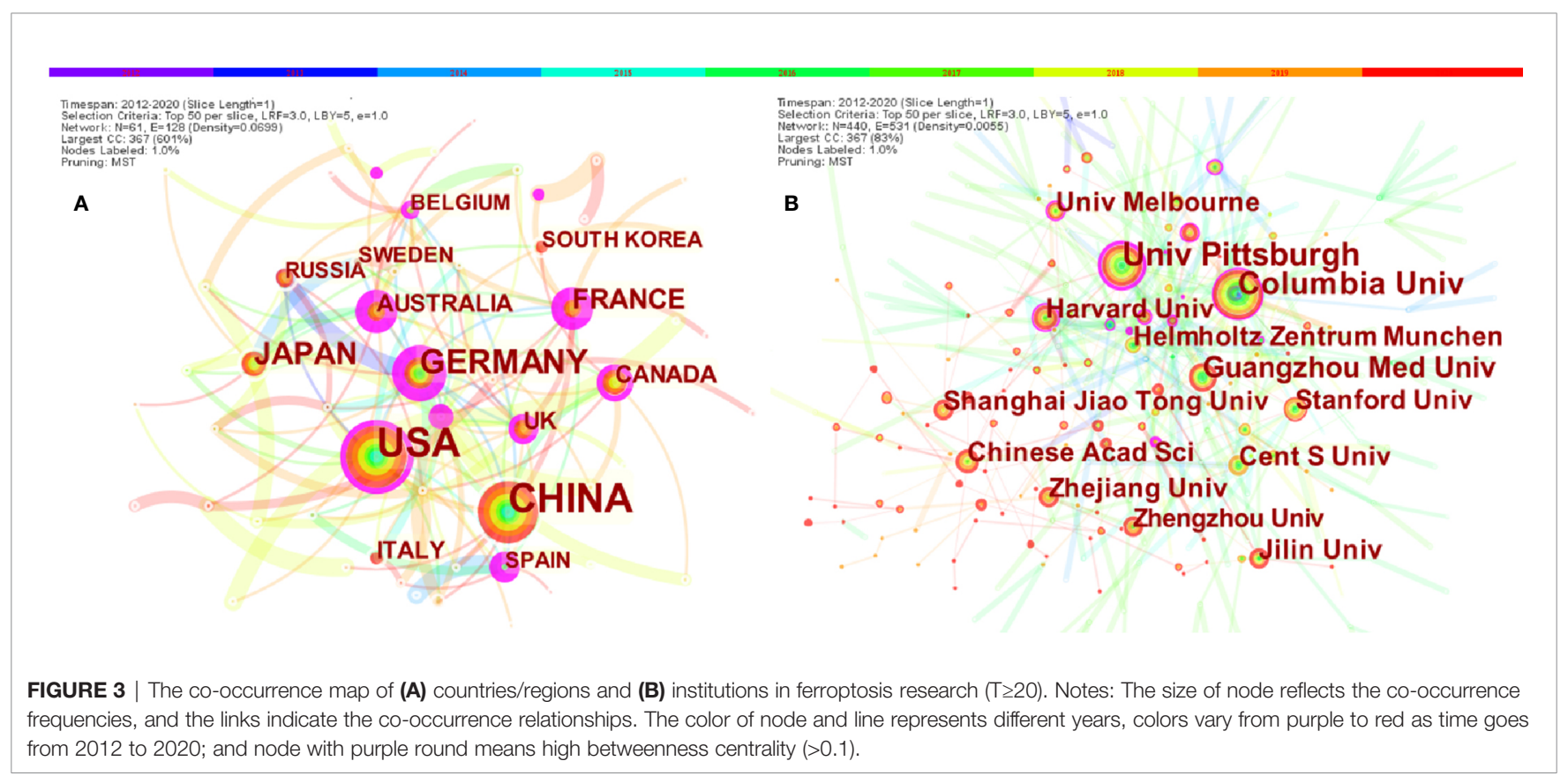


TABLE 4 | The top 10 authors and co-cited authors of ferroptosis research.

\begin{tabular}{llcc}
\hline Rank & \multicolumn{1}{c}{ Author } & Count & Co-cited author \\
1 & Brent R Stockwell & 39 & Scott J Dixon \\
2 & Daolin Tang & 36 & Wan Seok Yang \\
3 & Rui Kang & 35 & Jose Pedro Friedmann Angeli \\
4 & Marcus Conrad & 31,566 & Minghui Gao \\
5 & Andreas Linkermann & 26 & Andreas Linkermann \\
6 & Scott J Dixon & 22 & Brent R Stockwell \\
7 & Jose Pedro Friedmann Angeli & 14 & Sebastian Doll \\
8 & Valerian E Kagan & 13 & Lorenzo Galluzzi \\
9 & Guido Kroemer & 13 & Yangchun Xie \\
10 & Jiao Liu & 13 & Li Jiang \\
10 & Shinya Toyokuni & 13 & 362 \\
\end{tabular}

cited authors clearly. According to Figure 5, Scott J Dixon and Wan Seok Yang had the hottest color for the most co-cited.

\section{Keyword Co-Occurrence, Clusters, and Evolution}

VOSviewer was used to present the term co-occurrence (Table 5,

Figure 6, 7) and cluster analysis (Figure 7). A total of 25,413 terms were extracted, of which 595 appeared more than ten times and 51 appeared more than 100 times. The density map (Figure 6) of terms can find the high-frequency co-occurrence terms, which reveal the hotspots in a specific research field. As we can see from Figure 6 and Table 5, peroxidation was the most important term with 350 (2.97\%) co-occurrences, followed by disease, tumor cell, tumor, review and necroptosis.

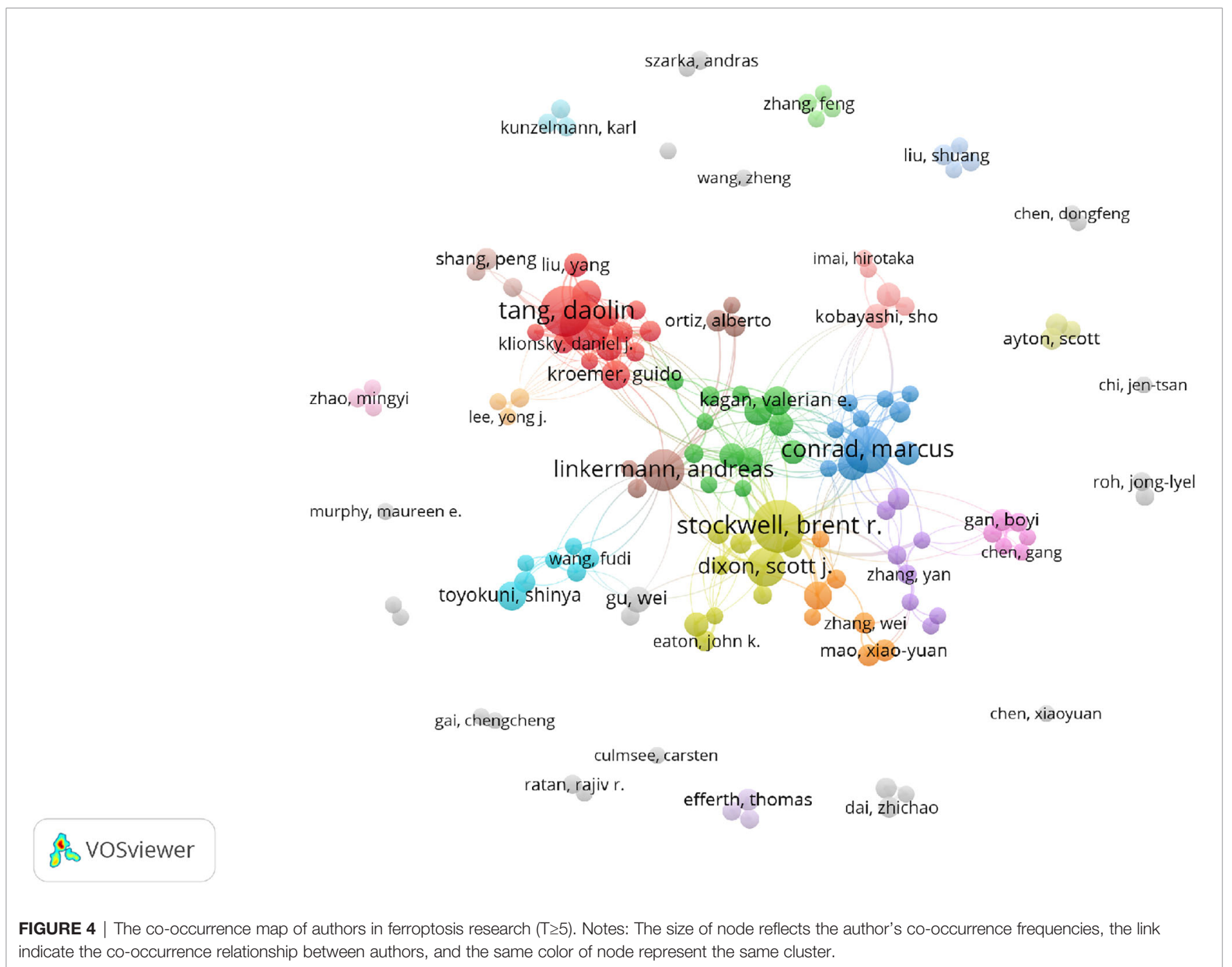




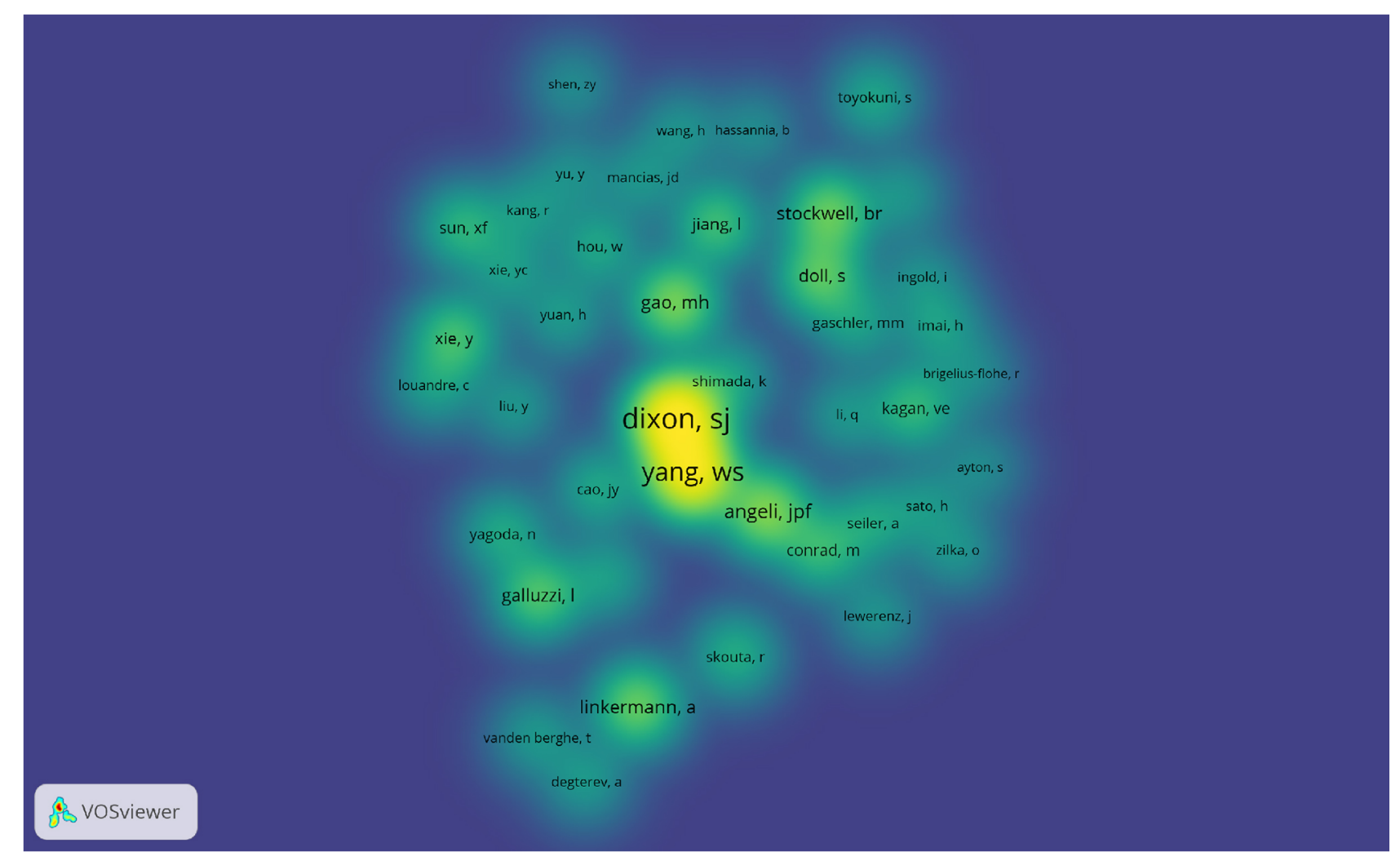

FIGURE 5 | The density map of co-cited authors in ferroptosis research ( $\geq 100)$. Notes: The size of word, the size of round, and the opacity of yellow is positively related to the co-cited frequency.

Cluster analysis can show the knowledge structure of the research field (29). According to the link strength of term cooccurrence, the network was divided into five clusters (Figure 7). It is highly homogeneous between the terms in one cluster. Cluster 1 (red) is the largest cluster with 110 co-occurrence terms: erastin, cell line, ferrostatin-1, cell viability, knockdown, cytotoxicity, cell viability, SLC7A11, malonaldehyde (MDA), sorafenib, caspase, ferrous iron, system xc-, RSL3, miRNA, siRNA, etc. The topic of Clusterl is the mechanism of ferroptosis. Cluster 2 (green) is mainly related to nervous system injury, which includes 73 terms: disease, neurodegeneration, dysfunction, pathogenesis, neuron, iron homeostasis, Parkinson's disease, Alzheimer, stroke, neuronal death, etc. Cluster 3 (blue) focuses on cancer, which contains 69 terms: tumor cell, tumor, anti-cancer therapy, peroxide, potential, application, chemotherapy, nanomaterial, new strategy, tumor microenvironment, photothermal therapy, photodynamic therapy, etc. Cluster 4 (yellow) is mainly related to cell death with 63 terms: review, necroptosis, necrosis, injury, inflammation, cell death pathway, pyroptosis, regulated necrosis,

TABLE 5 | The top 20 terms of ferroptosis research (relevance score>0.081).

\begin{tabular}{|c|c|c|c|c|c|}
\hline Rank & Term & Count & Rank & Term & Count \\
\hline 1 & peroxidation & 350 (2.97\%) & 12 & neurodegeneration & $106(0.90 \%)$ \\
\hline 2 & disease & $321(2.73 \%)$ & 13 & cancer therapy & $99(0.84 \%)$ \\
\hline 3 & tumor cell & 258 (2.19\%) & 13 & inflammation & 99 (0.84\%) \\
\hline 4 & tumor & 186 (1.58\%) & 15 & dysfunction & $96(0.82 \%)$ \\
\hline 5 & review & 185 (1.57\%) & 16 & peroxide & $92(0.78 \%)$ \\
\hline 6 & necroptosis & 168 (1.43\%) & 17 & potential & $88(0.75 \%)$ \\
\hline 7 & erastin & 148 (1.26\%) & 18 & cell death pathway & $86(0.73 \%)$ \\
\hline 8 & injury & $141(1.20 \%)$ & 19 & release & $85(0.72 \%)$ \\
\hline 9 & necrosis & $121(1.03 \%)$ & 20 & ferroptosis inhibitor & $84(0.71 \%)$ \\
\hline 10 & cell line & $113(0.96 \%)$ & 20 & pyroptosis & $84(0.71 \%)$ \\
\hline 11 & ferrostatin-1 & $111(0.94 \%)$ & 20 & sensitivity & $84(0.71 \%)$ \\
\hline
\end{tabular}




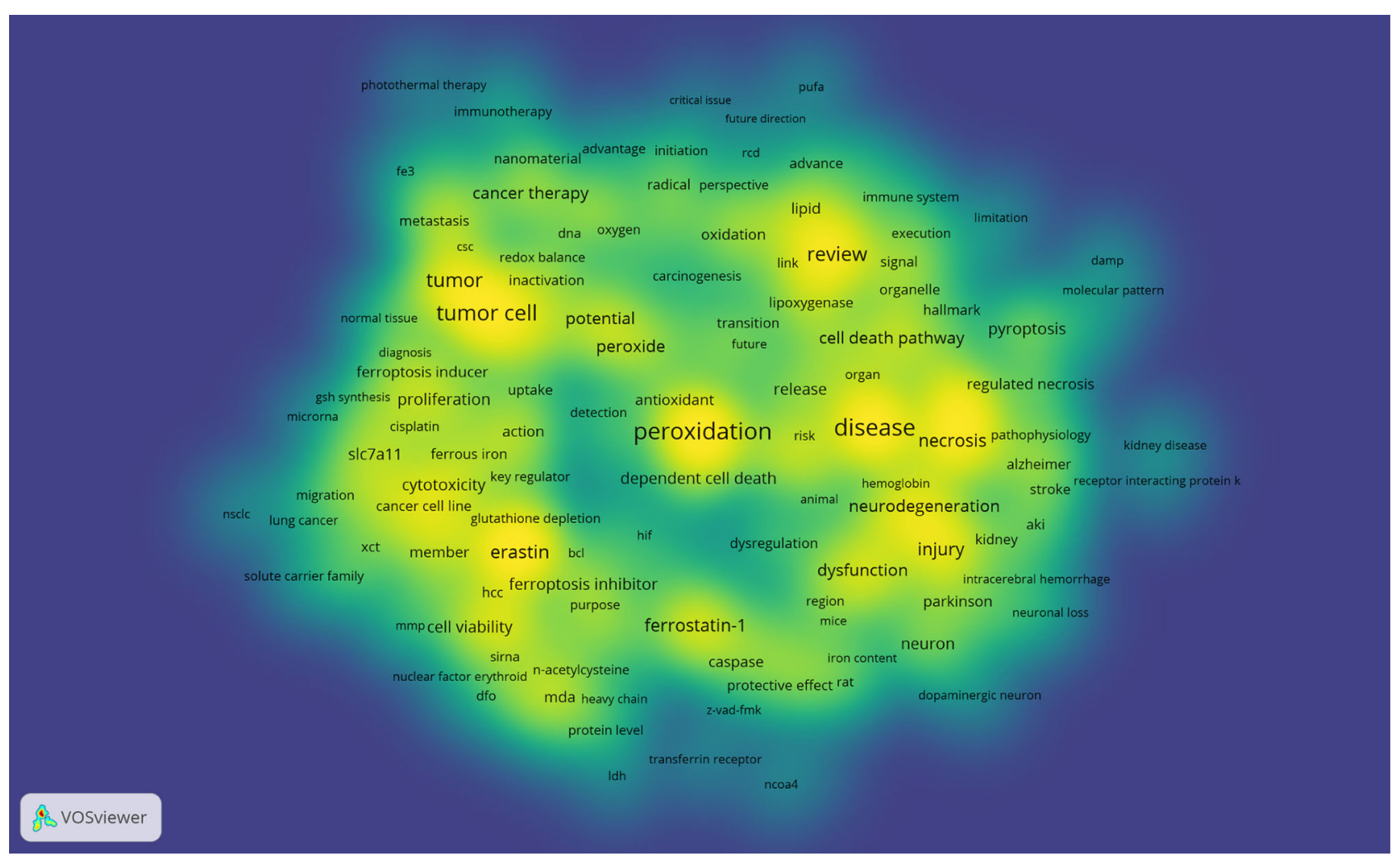

FIGURE 6 | The density map of terms in ferroptosis research $(T \geq 10$, relevance score $\geq 0.081)$ Notes: The size of word, the size of round, and the opacity of yellow is positively related to the co-occurrence frequency.

cell death mechanism, ischemia-reperfusion, acute kidney injury (AKI), etc. Cluster 5 (purple) is related to lipid peroxidation, which includes 42 terms: peroxidation, oxidation, lipid, antioxidant, phospholipid, lipoxygenase, lipid hydroperoxide, acyl-CoA synthetase long chain family member 4 (ACSL4), polyunsaturated fatty acid (PUFAs), etc.

Keywords time zone view was designed by CiteSpace, which could show the evolution of high-frequency keywords clearly. Keywords were located in the year they first co-occurred, and the color of links represents the first year two keywords appear simultaneously. High-frequency keywords $(T \geq 50)$ were shown in Figure 8, while the threshold was a cumulative figure, leading some latest keywords had not accumulated 50 enough. Consequently, we added the annual top three high-frequency keywords from 2016 to 2019 to supplement the timezone map (Figure 8). Among them, nuclear factor erythroid-2 related factor 2 (Nrf2) may have turning point significance with a high centrality (0.12) more than $0.10(41)$.

\section{Co-Cited Reference and Reference Burst}

We used CiteSpace to detect the co-cited references. Table 6 showed that the top 10 co-cited references were co-cited at least 196 times, especially three of them were co-cited over 300 times. The most co-cited reference was a review published in Cell by Brent R Stockwell, etc. in 2017 (1), entitled "Ferroptosis:
A Regulated Cell Death Nexus Linking Metabolism, Redox Biology, and Disease", followed by an article entitled "Regulation of ferroptotic cancer cell death by GPX4" (3).

References with citation bursts are defined as those that are cited frequently over a while (41). In CiteSpace, we set the burst duration to at least two years, from which we detected 55 references with the strongest citation bursts (Figure 9). Figure 9 showed that $34.55 \%(19 / 55)$ of the references appeared citation burstness in 2014, followed by $2016(15 / 55,27.27 \%)$ and 2015 $(7 / 55,12.73 \%)$. Notably, nine references (16.36\%) were in burstness until 2020. The paper with the strongest burstness (strength=79.99) was entitled "Ferroptosis: an iron-dependent form of non-apoptotic cell death" (2), published in Cell by Scott J Dixon, etc. in 2012, with citation burstness from 2013 to 2017.

\section{DISCUSSION}

\section{General Information}

Based on the data from WoSCC database up to November 1, 2020 , a total of 1,267 ferroptosis researches were published in 466 academic journals by 6,867 authors in 438 institutions from 61 countries/regions.

Change of the annual output is an essential indicator for the development trend in the field $(29,35)$. The ferroptosis research 


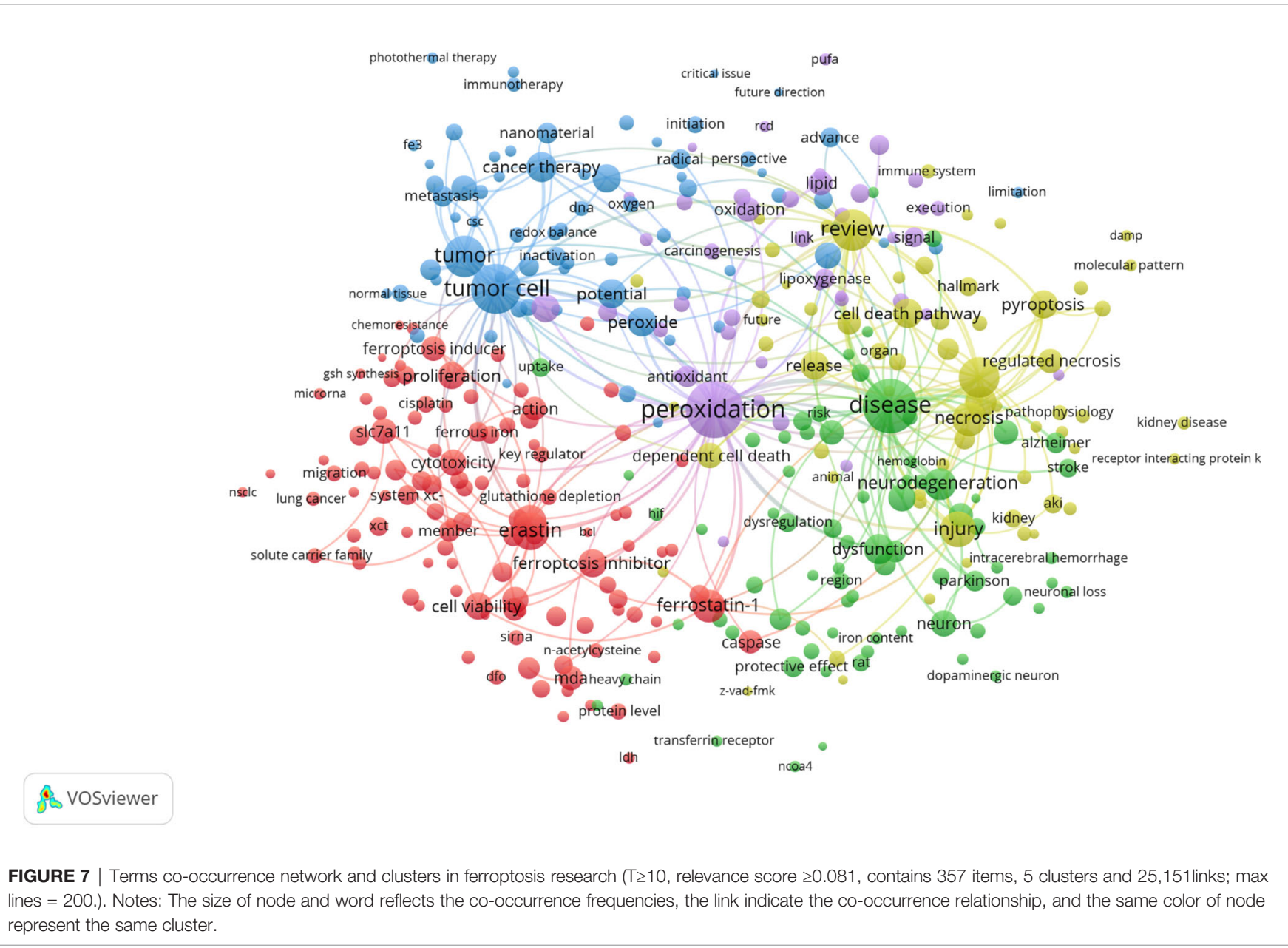

officially started in 2012, the year Scott J Dixon presented "ferroptosis" (2), and showed an upward tendency overall (Figure 1). It could be divided into three stages, namely, "Germination," "Stable growth," and "Rapid development." "Germination"(2012-2013): The concept of ferroptosis was officially proposed (2), and there were four articles in these 2 years. "Stable growth" (2014-2017): In this stage, ferroptosis gained more scientists' interest and the annual output grew steadily. "Rapid development" (2018 to present): During this period, the number of annual publications was approximately twice that of the previous year, indicating that ferroptosis research has attracted mounting researchers' attention and developed rapidly. Furthermore, the increasing trend looks promising.

Journals and co-cited journals analysis (Table 1) showed that Cell Death \& Disease published the most ferroptosis research, while Cell received the largest number of co-cited references. Both of these are journals on cell biology, which is consistent with the dual-map analysis (Figure 2). The dual-map overlay of journals stands for the topic distribution of academic journals (38); Figure 2 showed only one main citation path from Molecular/Biology/Genetics co-cited journals to Molecular/ Biology/Immunology journals, implying that ferroptosis-related studies are focused on basic research nowadays, while researches on translational medicine is still limited (7). Meanwhile, journals at the Q1 JCR division with high IF accounted for the majority of top 10 journals (70\%) and co-cited journals (80\%), suggesting that these journals have interests and play essential roles in ferroptosis-related researches.

Are there differences among countries/institutions in ferroptosis studies? Table 3 and Figure $\mathbf{3}$ showed that China, the USA, and Germany were the top 3 productive countries. However, the USA, France, Germany, Australia, the United Kingdom, and Canada were regarded as important turning points that may lead to transformative discoveries $(37,39-41)$. Furthermore, the United States was the earliest country to take up the ferroptosis study, followed by Germany, France, and Russia; these four countries were also the top 10 productive countries. Indicating that the United States is always a productive and influential country in ferroptosis research; noticeably, China started later but has emerged as one of the most productive contributors in recent years. That is consistent with the finding in neuroscience research, and may be related to the economic development and financial input into academic research of these countries (50). Besides, there were active collaborations among different countries/regions, especially the United States, indicating ferroptosis-related research had gained interest 


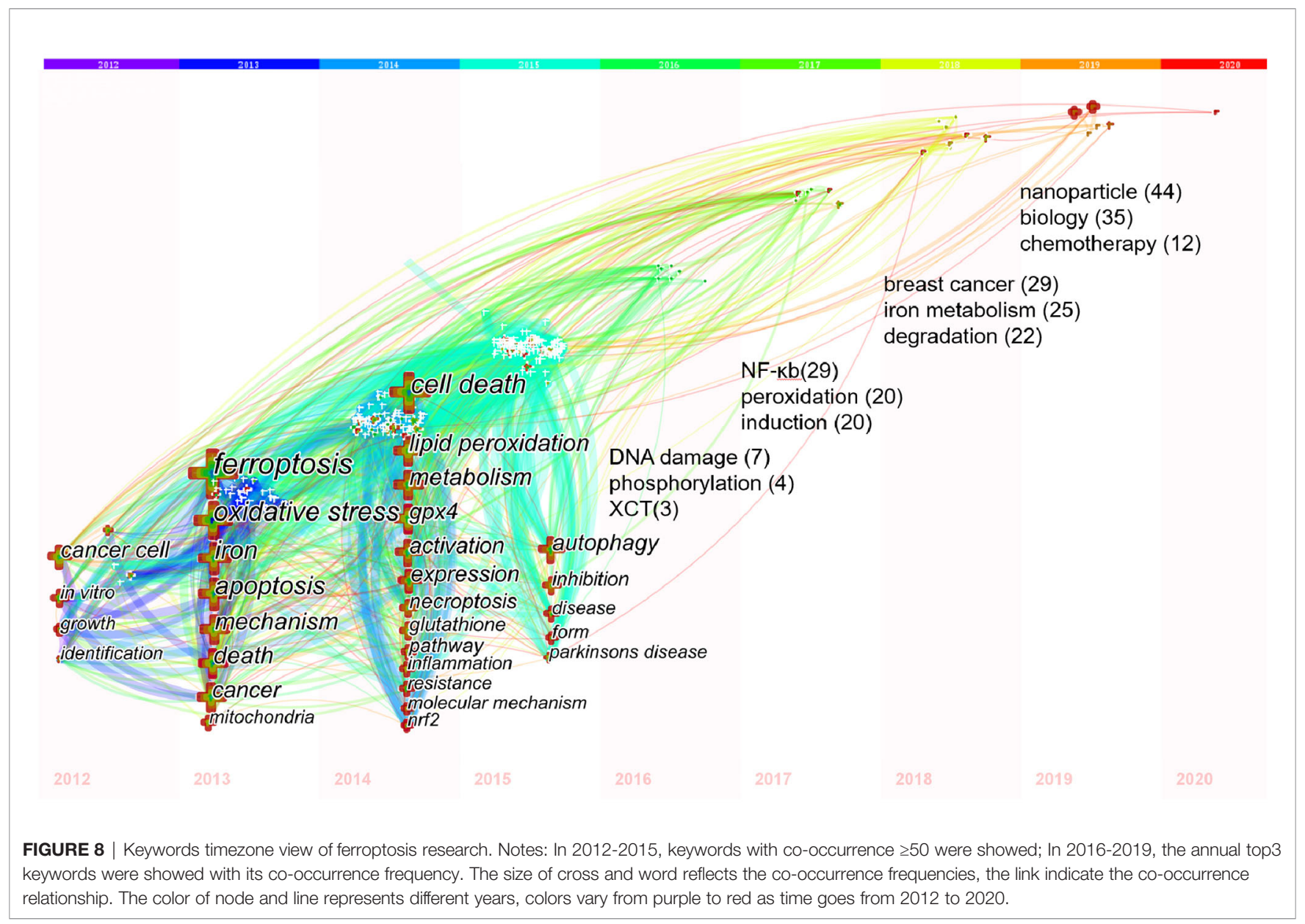

worldwide, and the United States was the main collaborating center. The top 12 institutions were from four countries; threefifths were from China, while the top 2 were from the USA. The University of Pittsburgh, Columbia University, and Guangzhou Medical University published the most. Moreover, we found active cooperation among the University of Pittsburgh, Columbia University, Harvard University, and other institutions, implying their notable contributions to the ferroptosis field.

Highlighting the contributions of influential researchers, such as the authors with many co-occurrences or co-cited papers in a specific field, can help scholars move along the road and provide further directions and guidelines (51). In our analysis (Table 4, Figures 4 and 5), Brent R Stockwell published the most papers, while Scott J Dixon had the most co-citations. Meanwhile, we found four scholars who were not only the top 10 productive authors but also the top 10 co-cited authors, namely Brent $\mathrm{R}$ Stockwell, Andreas Linkermann, Scott J Dixon, and Jose Pedro Friedmann Angeli. Implying that these four authors had an outstanding contribution to ferroptosis field. Furthermore, the map of authors and co-cited authors provides information about potential collaborators and influential research groups (52).

TABLE 6 | Top 10 co-cited references for ferroptosis research.

\begin{tabular}{|c|c|c|c|c|}
\hline Rank & ID & Title & Journal & Co-citation \\
\hline 1 & Stockwell BR (1) & Ferroptosis: A Regulated Cell Death Nexus Linking Metabolism, Redox Biology, and Disease & Cell & 433 \\
\hline 2 & Yang WS (3) & Regulation of ferroptotic cancer cell death by GPX4 & Cell & 374 \\
\hline 3 & Xie $Y(43)$ & Ferroptosis: process and function & Cell Death Differ & 335 \\
\hline 4 & Jiang L (44) & Ferroptosis as a p53-mediated activity during tumor suppression & Nature & 279 \\
\hline 5 & Doll S (45) & ACSL4 dictates ferroptosis sensitivity by shaping cellular lipid composition & Nat Chem Biol & 276 \\
\hline 6 & Yang WS (46) & Ferroptosis: Death by Lipid Peroxidation & Trends Cell Biol & 273 \\
\hline 7 & Angeli JPF (4) & Inactivation of the ferroptosis regulator Gpx4 triggers acute renal failure in mice & Nat Cell Biol & 271 \\
\hline 8 & Gao MH (47) & Glutaminolysis and Transferrin Regulate Ferroptosis & Mol Cell & 240 \\
\hline 9 & Kagan VE (48) & Oxidized arachidonic and adrenic PEs navigate cells to ferroptosis & Nat Chem Biol & 234 \\
\hline 10 & Yang WS (49) & Peroxidation of polyunsaturated fatty acids by lipoxygenases drives ferroptosis & Proc Natl Acad Sci U S A & 196 \\
\hline
\end{tabular}




\section{Top 55 References with the Strongest Citation Bursts}

\begin{tabular}{|c|}
\hline \\
\hline Fuchs Y, 2011, CELL, V147, P742, DOI 10.1016/..cell.2011.10.033, DOI \\
\hline Wolpaw As, 2011, P NATL ACAD SCI USA V108, P0, DOI 10.1073/pnas. 1106149108, DOI \\
\hline Dixon SJ, 2012, CELL, V149, P1060, DOI 10.1016/j.cell.2012.03.042, D0.] \\
\hline Galmiche A, 2010, MOL CANCER RES, V8, P1116, DOI 10.1158/1541-7786.MCR-10-0029, DOI \\
\hline Coriat $R, 2012$, MOL CANCER THER V11, P2284, DOI 10.1158/1535-7163.MCT-12-0093, DOI \\
\hline Louandre C, 2013, INT J CANCER, V133, P1732, DOI 10.1002/ijc.28159, DO|] \\
\hline Sun LM, 2012, CELL, V148, P213, DOI 10.1016/.j.ell.2011.11.031, DOI \\
\hline Linkermann A. 2013, P NATL ACAD SCI USA, V110, P12024, DOI 10.1073/pnas.1305538110, DOI \\
\hline Brigelius-Flohe R, 2013, BBA-GEN SUBJECTS, V1830, P3289, DOI 10.1016/.bbagen.2012.11.020, \\
\hline Vanden Berghe T, 2014, NAT REV MOL CELL BIO, V15, P134, DOI 10.1038/nrm3737, DOI \\
\hline Galluzzi L, 2012, CELL DEATH DIFFER, V19, P107, DOI 10.1038/cdd.2011.96, DOI \\
\hline Takahashi N, 2012, CELL DEATH DIS, V3, PO, DOI 10.1038/cddis.2012.176, DOI \\
\hline Oberst A, 2011, NATURE, V471, P363, DOI 10.1038/nature09852, DOl \\
\hline Linkermann A, 2012, KIDNEY INT, V81, P751, DOI 10.1038/ki.2011.450, DO \\
\hline Kaiser WJ, 2013, J BIOL CHEM, V288, P31268, DOI 10.1074/fbc.M113.462341, DOI \\
\hline Kaczmarek A, 2013, IMMUNITY, V38, P209, DOI 10.1016/j.jimmuni.2013.02.003, DOI \\
\hline Cai ZY, 2014, NAT CFLL BIOL, V16, P55, DOI 10.1038/ncb2883, DOI \\
\hline Upton JW, 2012, CELLL HOST MICROBE, V11, P290, DOI 10.1016/].chom.2012.01.016, DOI \\
\hline Wu JF, 2013, CELL RES, VE3, P994, DOI 10.1038/cr.2013.91, DOI \\
\hline Vandenabeele P, 2010, NAT REV MOL CELL BIO, V11, P700, DOI 10.1038/nrm2970, DOI \\
\hline Conrad M, 2012, AMIN ACIDS, V42, P231, DOI 10.1007/500726-011-0867-5, DOI \\
\hline Murphy IM, 2013, IMMUNITY, V39, P443, DOI 10.1016/j.jmmuni.2013.06.018, DOI \\
\hline Kaiser WJ, 2011, NATURE, V471, P368, DO 10.1038/nature09857, DOa \\
\hline Linkermann A, 2014, NEW ENGL J MED, V370, P455, DOI 10.1056/NEJMra1310050, DQI \\
\hline Wortmann M, 2013, CIRC RES, V113, P408, DOI 10.1161/CIRCRESAHA.113.279984, DOI \\
\hline Wang HY, 2014, MOL CELL, V54, P133, DOI 10.1016/j:molcel.2014.03.003, DOI \\
\hline Li TY, 2012, CELL, V149, P1269, DOI 10.1016/j.cell.2012.04.026, DOI \\
\hline Chen X, 2014, CELL RES, V24, P105, DOI 10.1038/cr.2013.171, DOI \\
\hline
\end{tabular}

\begin{tabular}{|c|c|}
\hline \multicolumn{2}{|c|}{ Year Strength Begin End 2012 - 2020} \\
\hline 2011 & $4.8220122016 \_$ \\
\hline 2011 & 3.6520122015 \\
\hline 2012 & 79.9920132017 _ \\
\hline 2010 & 2.4420132015 \\
\hline 2012 & 2.4420132015 \\
\hline 2013 & 13.2820142017 \\
\hline 2012 & $9.6120142017=$ \\
\hline 2013 & 8.6920142017 \\
\hline 12013 & 8.5220142018 \\
\hline 2014 & 7.9620142017 \\
\hline 2012 & 6.6120142016 \\
\hline 2012 & 6.0520142016 \\
\hline 2011 & 6.0520142016 \\
\hline 2012 & 5.520142016 \\
\hline 2013 & 5.520142016 \\
\hline 2013 & 4.9520142016 \\
\hline 2014 & 4.420142016 \\
\hline 2012 & $3.8520142016=$ \\
\hline 2013 & $3.8520142016 \ldots$ \\
\hline 2010 & $3.1120142015=$ \\
\hline 2012 & 3.1120142015 \\
\hline 2013 & $3.1120142015=$ \\
\hline 2011 & $3.1120142015=$ \\
\hline 2014 & $24920142015=$ \\
\hline 2013 & $6.2720152017 \ldots$ \\
\hline 2014 & $5.7620152016 \ldots$ \\
\hline 2012 & $5.1820152016=$ \\
\hline 2014 & 4.0320152016 \\
\hline
\end{tabular}

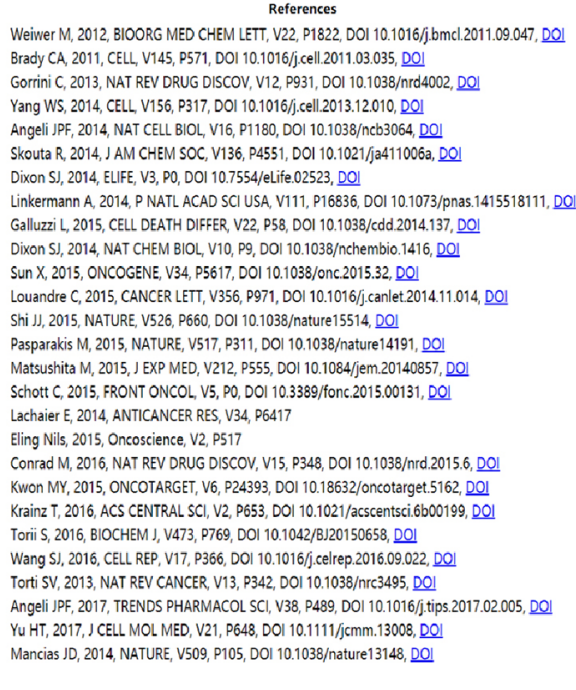

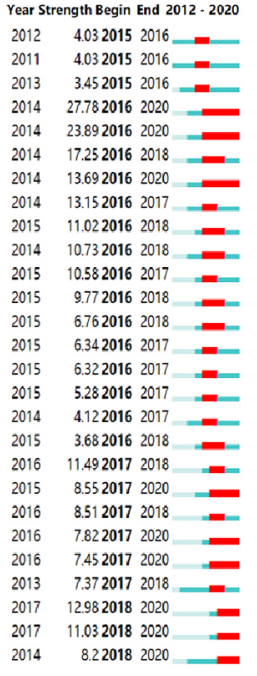

FIGURE 9 | Top 55 references with the strongest citation bursts (sorted by the beginning year of burst). Notes: The Blue bars mean the reference had been published; the red bars mean citation burstness.

In the ferroptosis field, researchers have active cooperation within and between institutions, especially among the influential authors. For example, 27 researchers from 24 institutions presented the most co-cited review entitled "Ferroptosis: A Regulated Cell Death Nexus Linking Metabolism, Redox Biology, and Disease" (1). It is suggesting that these influential teams could be potential collaborators for researchers.

\section{Knowledge Base}

Co-cited references are references that have been cited together by other publications. However, the knowledge base is the collection of co-cited references cited by the corresponding research community $(41,53-55)$, which is not entirely equivalent to highly cited references. In this bibliometric analysis, the top 10 references co-cited by the included ferroptosis literature (Table 6) were as follows.

In 2017, Cell published the most co-cited study $(n=430)$ co-authored by Brent R Stockwell and 26 other scholars (1) outstanding in ferroptosis research. This review summarized the mechanisms of ferroptosis, highlighted connections with other biological and medical areas, and recommended guidelines for studying ferroptosis. Wan Seok Yang et al. (3) published the second co-cited study in Cell in 2014. This study found GPX4 is an essential regulator of ferroptotic cancer cell death; before that, GPX4 had been proved to protect against lipid peroxidation (56) and oxidative stress damage (57). The third cocited publication was published in 2016 by Y Xie et al. (43) This review summarized the mechanisms, signaling pathways, and measuring methods of ferroptosis and discussed the role of ferroptosis in disease. The fourth co-cited paper was published by Le Jiang et al. (44) in Nature in 2015. This study showed that p53 inhibits cystine uptake and sensitizes cells to ferroptosis by repressing the expression of SLC7A11, a vital component of the cystine/glutamate antiporter. In 2017, Sebastian Doll et al. (45) established the essential role of ACSL4 in ferroptosis and published the fifth co-cited study. They used two approaches, genome-wide CRISPR-based genetic screen and microarray analysis of ferroptosis-resistant cell lines, to reveal that ACSL4 dictates ferroptosis sensitivity by shaping cellular lipid composition. The sixth co-cited paper was published by Wan Seok Yang et al. (46) in 2016. This review summarized the discovery of ferroptosis, the molecular mechanisms controlling ferroptosis, and its increasingly appreciated relevance to health and disease. Nature Cell Biology published the seventh co-cited experiment research by Jose Pedro Friedmann Angeli et al. in 2014 (4). This study used inducible Gpx4(-/-) mice to elucidate an essential role for the GSH/Gpx4 axis in preventing lipidoxidation-induced acute renal failure. Furthermore, they found Liproxstatin-1, a spiroquinoxalinamine derivative, is a potent ferroptosis inhibitor in cells, Gpx4(-/-) mice, and a pre-clinical model of ischemia/reperfusion-induced hepatic damage. The eighth co-cited paper was published by Minghui Gao et al. (47) in Molecular Cell in 2015. This study detected that the ironcarrier protein transferrin and amino acid glutamine are the inducers of ferroptosis; and glutaminolysis, the cell surface transferrin receptor and the glutamine-fueled intracellular metabolic pathway, plays crucial roles in the ferroptosis process. Furthermore, this study proved that inhibiting glutaminolysis can reduce ischemia/reperfusion-induced heart damage. In 2017, Nature Chemical Biology published the ninth co-cited study authored by Valerian E Kagan et al. (48). This study used quantitative redox lipidomics, reverse genetics, bioinformatics, and systems biology to detect the peroxidation 
mechanism of ferroptosis, and discovered that oxidized arachidonic and adrenic PEs navigate cells to ferroptosis, which may be useful to anti-cancer therapy. In 2016, the tenth co-cited paper was published by Wan Seok Yang et al. (49) in PNAS. They demonstrated that PUFAs are susceptible to lipid peroxidation by lipoxygenases and hence execute ferroptosis.

Generally, the top 10 co-cited references focused on reviews (three reviews were published in 2017 and 2016), mechanisms (include targets and genes, such as GPX4, glutamine, GSH, ironcarrier protein transferrin, p53, SLC7A11, ACSL4, system xc-, PUFAs, lipoxygenase, etc.), and related diseases of ferroptosis (such as cancers, acute renal failure, ischemia/reperfusioninduced hepatic and heart damage etc.), all these were the foundations of ferroptosis research.

\section{Hotspot Evolution, Knowledge Structure, and Emerging Topics}

In bibliometrics, keywords/terms co-occurrence (Table 5 and Figure 6) can reflect the hotspots of an academic field (58), and the timezone view (Figure 8) can show the evolution of new hotspots (59). The high-frequency terms of ferroptosis (Table 5 and Figure 6) included peroxidation, inflammation, disease, tumor, cancer therapy, neurodegeneration, review, necroptosis, pyroptosis, etc., which were regarded as the hotspots in ferroptosis research. As time goes on, emerging topics occurred continuously (Figure 8). In the germination stage (2012-2013), rising terms included cancer cell, oxidative, mechanism, mitochondria, etc. While in the stable-growth stage (20142017), new terms contained more mechanism detection and pay more attention to different diseases, including lipid peroxidation, GPX4, glutathione, pathway, inflammation, resistance, molecule mechanism, Nrf2, disease, Parkinson's disease, DNA damage, nuclear factor kappa-B (NF- $\mathrm{kb}$ ), peroxidation, induction, etc. Notably, Nrf2 may have turning significance with a high centrality $(41,60)$. In the rapid development stage (2018-now), emerging topics, such as breast cancer, iron metabolism, degradation, nanoparticle, biology, did not only continue the characteristics of the stable-growth stage but also used more technology, such as the emerging application of nanoparticles in cancer treatment (61-67). Unfortunately, although many essential regulatory molecules were demonstrated and transferrin receptor 1 protein (TfR1) was considered as a specific marker of ferroptosis by some scholars (68), there are still no acknowledged specific biomarkers of ferroptosis, such as caspase activation for apoptosis or autophagy lysosome formation for autophagy $(9,69)$.

Moreover, the cluster of keywords/terms could describe the internal knowledge structure and reveal the research frontier of the discipline (29). Cluster analysis showed five main clusters in the ferroptosis field (Figure 7), including regulation mechanisms, nervous system injury (including neurodegenerative disease), cancer, the relationship with other types of cell death, and lipid peroxidation, representing five main aspects of ferroptosis research to some extent. As we know, there is cross-talk between ferroptosis and other types of cell death in some similar signals and molecular regulators [e.g., apoptosis (70) and autophagy (71)], while the mechanisms that direct cells to choose among different cell death ways are still an enigma (7). Besides, lipid peroxidation is proved to be a vital mechanism in ferroptosis, but it is still unknown why and how lipid peroxidation leads to the death of cells in ferroptosis, and this is regarded as one of three key areas of future ferroptosis research (72). As for disease, ferroptosis in cancers has been a hotspot from initial stage to now, and there is also a large amount of research on nervous system injury.

References with strong citation bursts (Figure 9) could also characterize the emerging topics of a field $(35,41,73)$. The strongest citation burstness came from a landmark study published by Dixon SJ et al. (2) in 2012 (79.30, 2013-2017), which coined the term ferroptosis. Early research only recognized that cysteine is necessary to maintain the biosynthesis of glutathione and inhibit a type of cell death in mammalian cells $(74,75)$ that is also preventable by iron chelators or lipophilic antioxidants (76). While in this study, Dixon SJ's team found that erastin could trigger a unique iron-dependent form of nonapoptotic cell death and named it ferroptosis. Since then, additional compounds and regulatory mechanisms have been identified, and ferroptosis becomes an emerging focus of regulated cell death. More importantly, among the top 55 references with the strongest citation burst (Figure 9), nine references are still in burstness. These nine references represent the latest emerging topics of ferroptosis hence they deserve further discussion $(19,35)$. Ranking by burstness strength, the first paper (strength $=27.78$ ) was published by Wan Seok Yang et al. (3) in Cell in 2014, with the citation burstness lasted for five years (20162020), proving that GPX4 is an essential regulator of ferroptotic cancer cell death. Jose Pedro Friedmann Angeli et al. (4) detected that inactivation of Gpx4 triggers acute renal failure in mice. The study was published in Nature Cell Biology in 2014 with the second strongest citation burstness (strength=23.89) lasting for five years (2016-2020). Scott J Dixon et al. (77) published the third reference in $2014(13.69,2016-2020)$. They found that erastin is a potent inhibitor of system $\mathrm{xc}$ - function, which is much more potent than sulfasalazine, the known best inhibitor of system xc-. They also discovered that the anti-cancer drug sorafenib inhibits system xcfunction and could trigger endoplasmic reticulum stress and ferroptosis. The reference with the fourth-strongest citation burstness was published in Trends in Pharmacological Science by Jose Pedro Friedmann Angeli et al. (78) in 2017 (12.98, 20182020). This review mainly summarized the fundamental aspects of lipid peroxidation in ferroptosis and their potential contribution to disease; furthermore, they discussed the potential pharmacological approaches aiming to subvert lipid peroxidation and suppress ferroptosis. The fifth paper is a review authored by Haitao $\mathrm{Yu}$ et al. (79) in 2017 (11.03, 2018-2020), focusing on the relationship between ferroptosis and human tumorous diseases. Min-Young Kwon et al. (80) published the sixth study in 2015 (8.55, 20172020). They elucidated that heme oxygenase-1 (HO-1) accelerates erastin-induced lipid peroxidation and ferroptosis. The seventh reference (81) was published in Nature in 2014 (8.2, 2018-2020). This work identified nuclear receptor coactivator 4 (NCOA4) as a selective cargo receptor for autophagic turnover of ferritin. In previous studies, genetic overexpression or knockdown of NCOA4 has been shown to trigger or prevent erastin-induced 
ferroptosis in several (82). Indeed, ferroptosis has been suggested as a type of autophagy-dependent cell death (71). The eighth study (83) (7.82, 2017-2020) detected that functional lysosomes play an essential role in functional lysosomes in the ferroptosis of cancer cells. Moreover, the ninth study (84) (7.45, 2017-2020) demonstrated the vital role of $\mathrm{p} 53$ acetylation in ferroptosis and its remaining tumor suppression activity. The citation burstness analysis showed that exploring the mechanism of ferroptosis (such as GPX4, lipid peroxidation, $\mathrm{HO}-1$, NCOA4, functional lysosomes, p53 acetylation, etc.) and applying to related disease (such as tumor, acute renal failure, etc.) were the recent major topics in the field of ferroptosis research.

From the above analysis, we can see that ferroptosis research initially focused on experimental research and cancer. Afterward, in-depth experimental research detected more star mechanisms (such as GPX4, Nrf2, GSH, p53, SLC7A11, ACSL4, system xc-, phospholipids, $\mathrm{NAD}(\mathrm{P}) \mathrm{H}$, CoQ10, lipid peroxidation, etc.), used new technologies, and related ferroptosis to more diseases (such as kinds of cancer, Alzheimer, Parkinson's disease, stroke, ischemia-reperfusion damage, kidney disease, liver disease, atherosclerosis, drug resistance, etc.). Furthermore, ferroptosis research began to do clinical trials (85), while there are still no human intervention trials, indicating that the clinical translational application of ferroptosis theories is still ongoing (12). The reason might be that the ferroptosis inhibitors such as ferrostatin-1 and liproxstatin-1 may inhibit other ROSdependent forms of cell death, although they are safe in preclinical animal studies (7). In the latest traditional review, Daolin Tang (7) pointed out that we are at the dawn of ferroptosis research; challenges require more specific drugs, sophisticated pre-clinical models, and innovative technology. That is consistent with our bibliometric detecting.

\section{Limitations}

This study also comes with certain limitations inherent in bibliometrics. Firstly, data were retrieved only from the WoSCC database, while a few studies not included in WoSCC were missed. However, WoSCC is the most commonly applied database for scientometric analysis $(19,28)$; data from WoSCC could represent most information in a degree. Secondly, all information was extracted by bibliometric tools basing on machine learning and natural language processing, which may lead to bias as reported in other bibliometric studies (86). Nevertheless, compared to the latest traditional reviews $(7,9,72,87,88)$, our results are basically consistent with them while providing researchers with richer objective information, knowledge and insight.

\section{CONCLUSION}

In conclusion, ferroptosis research is in a rapid development stage with active cooperation worldwide, of which the United States is the main collaborating center. Current publications are mainly in the molecular and biology field. Five main aspects of ferroptosis research included regulation mechanisms, nervous system injury, cancer, relationships with other types of cell death, and lipid peroxidation. The latest hotspots are nanoparticle, cancer therapy, iron metabolism, and in-depth mechanism. Notably, Nrf2 may have turning significance. Based on the results, the emerging topics would be the further regulatory mechanism of ferroptosis and the broader application of ferroptosis-related disease with advanced technology.

Overall, this is the first study to systematically analyze the ferroptosis-related publications by bibliometric and knowledgemap. Moreover, we analyzed data by both CiteSpace and VosViewer, which could obtain richer results from different perspectives. Compared to traditional reviews, this study provides an original and objective insight into ferroptosis research. We believe the results of this study would provide helpful references for further research.

\section{AUTHOR'S NOTE}

The data used for analysis is in Annexes 1, and the result data is in Annexes 2.

\section{DATA AVAILABILITY STATEMENT}

The original contributions presented in the study are included in the article/Supplementary Material. Further inquiries can be directed to the corresponding authors.

\section{AUTHOR CONTRIBUTIONS}

HX and JJ designed this study. YF collected the data. JZ and LX performed the analysis. TW and WT normalized the pictures. JZ and LS wrote the original draft. All authors contributed to the article and approved the submitted version.

\section{FUNDING}

The work was supported by the National Natural Science Foundation of China (No. 81874412), National Natural Science Foundation of China (No.82004145), Beijing Natural Science Foundation (No.7204298), and Central Public Welfare Research Institutes of China Academy of Chinese Medical Sciences (No. ZZ13-YQ-017).

\section{ACKNOWLEDGMENTS}

The authors would like to thank National Clinical Research Center for Chinese Medicine Cardiology for supporting that work.

\section{SUPPLEMENTARY MATERIAL}

The Supplementary Material for this article can be found online at: https://www.frontiersin.org/articles/10.3389/fonc.2021. 686726/full\#supplementary-material. 


\section{REFERENCES}

1. Stockwell BR, Friedmann Angeli JP, Bayir H, Bush AI, Conrad M, Dixon SJ, et al. Ferroptosis: A Regulated Cell Death Nexus Linking Metabolism, Redox Biology, and Disease. Cell (2017) 171(2):273-85. doi: 10.1016/j.cell.2017. 09.021

2. Dixon SJ, Lemberg KM, Lamprecht MR, Skouta R, Zaitsev EM, Gleason CE, et al. Ferroptosis: An Iron-Dependent Form of Nonapoptotic Cell Death. Cell (2012) 149(5):1060-72. doi: 10.1016/j.cell.2012.03.042

3. Yang WS, SriRamaratnam R, Welsch ME, Shimada K, Skouta R, Viswanathan VS, et al. Regulation of Ferroptotic Cancer Cell Death by GPX4. Cell (2014) 156(1-2):317-31. doi: 10.1016/j.cell.2013.12.010

4. Friedmann Angeli JP, Schneider M, Proneth B, Tyurina YY, Tyurin VA, Hammond VJ, et al. Inactivation of the Ferroptosis Regulator Gpx4 Triggers Acute Renal Failure in Mice. Nat Cell Biol (2014) 16(12):1180-91. doi: $10.1038 /$ ncb3064

5. Yagoda N, von Rechenberg M, Zaganjor E, Bauer AJ, Yang WS, Fridman DJ, et al. Ras-RAF-MEK-Dependent Oxidative Cell Death Involving VoltageDependent Anion Channels. Nature (2007) 447(7146):864-8. doi: 10.1038/ nature05859

6. Vanden Berghe T, Linkermann A, Jouan-Lanhouet S, Walczak H, Vandenabeele P. Regulated Necrosis: The Expanding Network of NonApoptotic Cell Death Pathways. Nat Rev Mol Cell Biol (2014) 15(2):135-47. doi: $10.1038 / \mathrm{nrm} 3737$

7. Tang D, Chen X, Kang R, Kroemer G. Ferroptosis: Molecular Mechanisms and Health Implications. Cell Res (2021) 31(2):107-25. doi: 10.1038/s41422020-00441-1

8. Ingold I, Berndt C, Schmitt S, Doll S, Poschmann G, Buday K, et al. Selenium Utilization by GPX4 Is Required to Prevent HydroperoxideInduced Ferroptosis. Cell (2018) 172(3):409-22.e21. doi: 10.1016/j.cell.2017. 11.048

9. Li J, Cao F, Yin HL, Huang ZJ, Lin ZT, Mao N, et al. Ferroptosis: Past, Present and Future. Cell Death Dis (2020) 11(2):88. doi: 10.1038/s41419-020-2298-2

10. Wang H, Cheng Y, Mao C, Liu S, Xiao D, Huang J, et al. Emerging Mechanisms and Targeted Therapy of Ferroptosis in Cancer. Mol Ther (2021). doi: 10.1016/j.ymthe.2021.03.022

11. El Hout M, Dos Santos L, Hamaï A, Mehrpour M. A Promising New Approach to Cancer Therapy: Targeting Iron Metabolism in Cancer Stem Cells. Semin Cancer Biol (2018) 53:125-38. doi: 10.1016/j.semcancer.2018.07.009

12. Conrad M, Lorenz SM, Proneth B. Targeting Ferroptosis: New Hope for AsYet-Incurable Diseases. Trends Mol Med (2021) 27(2):113-22. doi: 10.1016/ j.molmed.2020.08.010

13. Jin Y, Zhuang Y, Liu M, Che J, Dong X. Inhibiting Ferroptosis: A Novel Approach for Stroke Therapeutics. Drug Discovery Today (2021) 26(4):91630. doi: 10.1016/j.drudis.2020.12.020

14. Wu X, Li Y, Zhang S, Zhou X. Ferroptosis as a Novel Therapeutic Target for Cardiovascular Disease. Theranostics (2021) 11(7):3052-9. doi: 10.7150/ thno. 54113

15. Chen X, Kang R, Kroemer G, Tang D. Broadening Horizons: The Role of Ferroptosis in Cancer. Nat Rev Clin Oncol (2021) 18(5):280-96. doi: 10.1038/ s41571-020-00462-0

16. Zheng J, Conrad M. The Metabolic Underpinnings of Ferroptosis. Cell Metab (2020) 32(6):920-37. doi: 10.1016/j.cmet.2020.10.011

17. Jiang X, Stockwell BR, Conrad M. Ferroptosis: Mechanisms, Biology and Role in Disease. Nat Rev Mol Cell Biol (2021) 22(4):266-82. doi: 10.1038/s41580020-00324-8

18. Forteza CM, Lunn E, Merigó JM, Horrach P. Research Progress in Tourism, Leisure and Hospitality in Europe (1969-2018). Int J Contemp Hospital Manage (2020) 33(1):48-74. doi: 10.1108/IJCHM-06-2020-0521

19. Ke L, Lu C, Shen R, Lu T, Ma B, Hua Y. Knowledge Mapping of Drug-Induced Liver Injury: A Scientometric Investigation (2010-2019). Front Pharmacol (2020) 11:842. doi: 10.3389/fphar.2020.00842

20. de Castilhos Ghisi N, Zuanazzi NR, Fabrin TMC, Oliveira EC. Glyphosate and its Toxicology: A Scientometric Review. Sci Total Environ (2020) 733:139359. doi: 10.1016/j.scitotenv.2020.139359

21. Chen C, Song M. Visualizing a Field of Research: A Methodology of Systematic Scientometric Reviews. PloS One (2019) 14(10):e0223994. doi: 10.1371/journal.pone.0223994
22. Ma C, Su H, Li H. Global Research Trends on Prostate Diseases and Erectile Dysfunction: A Bibliometric and Visualized Study. Front Oncol (2020) 10:627891. doi: 10.3389/fonc.2020.627891

23. Devos P, Ménard J. Trends in Worldwide Research in Hypertension Over the Period 1999-2018: A Bibliometric Study. Hypertension (2020) 76(5):1649-55. doi: 10.1161/hypertensionaha.120.15711

24. Chen C, Lou Y, Li XY, Lv ZT, Zhang LQ, Mao W. Mapping Current Research and Identifying Hotspots on Mesenchymal Stem Cells in Cardiovascular Disease. Stem Cell Res Ther (2020) 11(1):498. doi: 10.1186/s13287-02002009-7

25. Huang X, Fan X, Ying J, Chen S. Emerging Trends and Research Foci in Gastrointestinal Microbiome. J Transl Med (2019) 17(1):67. doi: 10.1186/ s12967-019-1810-x

26. Zhu X, Kong Q, Niu X, Chen L, Ge C. Mapping Intellectual Structure and Research Performance for the Nanoparticles in Pancreatic Cancer Field. Int J Nanomed (2020) 15:5503-16. doi: 10.2147/ijn.S253599

27. Zhang XL, Zheng Y, Xia ML, Wu YN, Liu XJ, Xie SK, et al. Knowledge Domain and Emerging Trends in Vinegar Research: A Bibliometric Review of the Literature From Woscc. Foods (2020) 9(2):166. doi: 10.3390/ foods 9020166

28. Mulet-Forteza C, Genovart-Balaguer J, Mauleon-Mendez E, Merigó JM. A Bibliometric Research in the Tourism, Leisure and Hospitality Fields. J Business Res (2019) 101:819-27. doi: 10.1016/j.jbusres.2018.12.002

29. Qin Y, Zhang Q, Liu Y. Analysis of Knowledge Bases and Research Focuses of Cerebral Ischemia-Reperfusion From the Perspective of Mapping Knowledge Domain. Brain Res Bull (2020) 156:15-24. doi: 10.1016/j.brainresbull. 2019.12.004

30. Merigó JM, Mulet-Forteza C, Valencia C, Lew AA. Twenty Years of Tourism Geographies : A Bibliometric Overview. Tourism Geograph (2019) 21(5):881910. doi: 10.1080/14616688.2019.1666913

31. Chen L, Ma S, Hu D, Lin H, Zhu Y, Chen K, et al. Bibliometric Study of Sodium Glucose Cotransporter 2 Inhibitors in Cardiovascular Research. Front Pharmacol (2020) 11:561494. doi: 10.3389/fphar.2020.561494

32. van Eck NJ, Waltman L. Software Survey: VOSviewer, A Computer Program for Bibliometric Mapping. Scientometrics (2010) 84(2):523-38. doi: 10.1007/ s11192-009-0146-3

33. Eck N, Waltman L. Vosviewer Manual. Leiden: Leiden University (2020).

34. Paunkov A, Chartoumpekis DV, Ziros PG, Sykiotis GP. A Bibliometric Review of the Keap1/Nrf2 Pathway and Its Related Antioxidant Compounds. Antioxid (Basel) (2019) 8(9):353. doi: 10.3390/antiox8090353

35. Gao Y, Shi S, Ma W, Chen J, Cai Y, Ge L, et al. Bibliometric Analysis of Global Research on PD-1 and PD-L1 in the Field of Cancer. Int Immunopharmacol (2019) 72:374-84. doi: 10.1016/j.intimp.2019.03.045

36. Forteza CM, Balaguer JG, Merigó JM, Mendez EM. Bibliometric Structure of IJCHM in its 30 Years. Int J Contemp Hospital Manage (2019) 31(12):4574604. doi: 10.1108/IJCHM-10-2018-0828

37. Chaomei C. Searching for Intellectual Turning Points: Progressive Knowledge Domain Visualization. Proc Natl Acad Sci USA (2004) 101(Suppl 1):5303-10. doi: 10.1073/pnas.0307513100

38. Chen C, Leydesdorff L. Patterns of Connections and Movements in Dual-Map Overlays: A New Method of Publication Portfolio Analysis. J Assoc Inf Sci Technol (2014) 65(2):334-51. doi: 10.1002/asi.22968

39. Chen C, Hu Z, Liu S, Tseng H. Emerging Trends in Regenerative Medicine: A Scientometric Analysis in Citespace. Expert Opin Biol Ther (2012) 12(5):593608. doi: 10.1517/14712598.2012.674507

40. Chen C. Searching for Intellectual Turning Points: Progressive Knowledge Domain Visualization. Proc Natl Acad Sci USA (2004) 101 Suppl 1(Suppl 1):5303-10. doi: 10.1073/pnas.0307513100

41. Chen C. Science Mapping:A Systematic Review of the Literature. J Data Inf Sci (2017) 2(02):1-40. doi: 10.1515/jdis-2017-0006

42. Li C, Wu K, Wu J. A Bibliometric Analysis of Research on Haze During 2000 2016. Environ Sci Pollut Res Int (2017) 24(32):24733-42. doi: 10.1007/s11356017-0440-1

43. Xie Y, Hou W, Song X, Yu Y, Huang J, Sun X, et al. Ferroptosis: Process and Function. Cell Death Differ (2016) 23(3):369-79. doi: 10.1038/cdd.2015.158

44. Jiang L, Kon N, Li T, Wang SJ, Su T, Hibshoosh H, et al. Ferroptosis as a p53Mediated Activity During Tumour Suppression. Nature (2015) 520(7545):5762. doi: $10.1038 /$ nature 14344 
45. Doll S, Proneth B, Tyurina YY, Panzilius E, Kobayashi S, Ingold I, et al. ACSL4 Dictates Ferroptosis Sensitivity by Shaping Cellular Lipid Composition. Nat Chem Biol (2017) 13(1):91-8. doi: 10.1038/nchembio.2239

46. Yang WS, Stockwell BR. Ferroptosis: Death by Lipid Peroxidation. Trends Cell Biol (2016) 26(3):165-76. doi: 10.1016/j.tcb.2015.10.014

47. Gao M, Monian P, Quadri N, Ramasamy R, Jiang X. Glutaminolysis and Transferrin Regulate Ferroptosis. Mol Cell (2015) 59(2):298-308. doi: 10.1016/j.molcel.2015.06.011

48. Kagan VE, Mao G, Qu F, Angeli JP, Doll S, Croix CS. Oxidized Arachidonic and Adrenic PEs Navigate Cells to Ferroptosis. Nat Chem Biol (2017) 13 (1):81-90. doi: $10.1038 /$ nchembio. 2238

49. Yang WS, Kim KJ, Gaschler MM, Patel M, Shchepinov MS, Stockwell BR. Peroxidation of Polyunsaturated Fatty Acids by Lipoxygenases Drives Ferroptosis. Proc Natl Acad Sci USA (2016) 113(34):E4966-75. doi: 10.1073/ pnas. 1603244113

50. Yeung AW, Goto TK, Leung WK. The Changing Landscape of Neuroscience Research, 2006-2015: A Bibliometric Study. Front Neurosci (2017) 11:120. doi: $10.3389 /$ fnins.2017.00120

51. Kodonas K, Fardi A, Gogos C, Economides N. Scientometric Analysis of Vital Pulp Therapy Studies. Int Endod J (2021) 54(2):220-30. doi: 10.1111/iej.13422

52. Liang YD, Li Y, Zhao J, Wang XY, Zhu HZ, Chen XH. Study of Acupuncture for Low Back Pain in Recent 20 Years: A Bibliometric Analysis Via Citespace. J Pain Res (2017) 10:951-64. doi: 10.2147/jpr.S132808

53. Chaomei C. Citespace II: Detecting and Visualizing Emerging Trends and Transient Patterns in Scientific Literature. J Am Soc Inf Sci Technol (2005) 57 (3):359-77. doi: 10.1002/asi.20317

54. Lu C, Liu M, Shang W, Yuan Y, Li M, Deng X, et al. Knowledge Mapping of Angelica Sinensis (Oliv.) Diels (Danggui) Research: A Scientometric Study. Front Pharmacol (2020) 11:294. doi: 10.3389/fphar.2020.00294

55. Chen C, Song M. Science Mapping Tools and Applications, In Representing Scientific Knowledge: The Role of Uncertainty. Springer (2017). p. 57-137.

56. Aitken RJ, Clarkson JS. Cellular Basis of Defective Sperm Function and its Association With the Genesis of Reactive Oxygen Species by Human Spermatozoa. J Reprod Fertil (1987) 81(2):459-69. doi: 10.1530/jrf.0.0810459

57. Yant LJ, Ran Q, Rao L, Van Remmen H, Shibatani T, Belter JG, et al. The Selenoprotein GPX4 is Essential for Mouse Development and Protects From Radiation and Oxidative Damage Insults. Free Radic Biol Med (2003) 34 (4):496-502. doi: 10.1016/s0891-5849(02)01360-6

58. Xiao F, Li C, Sun J, Zhang L. Knowledge Domain and Emerging Trends in Organic Photovoltaic Technology: A Scientometric Review Based on CiteSpace Analysis. Front Chem (2017) 5:67. doi: 10.3389/fchem.2017.00067

59. Liu G, Jiang R, Jin Y. Sciatic Nerve Injury Repair: A Visualized Analysis of Research Fronts and Development Trends. Neural Regen Res (2014) 9 (18):1716-22. doi: 10.4103/1673-5374.141810

60. Anandhan A, Dodson M, Schmidlin CJ, Liu P, Zhang DD. Breakdown of an Ironclad Defense System: The Critical Role of NRF2 in Mediating Ferroptosis. Cell Chem Biol (2020) 27(4):436-47. doi: 10.1016/j.chembiol.2020.03.011

61. Davis ME, Chen ZG, Shin DM. Nanoparticle Therapeutics: An Emerging Treatment Modality for Cancer. Nat Rev Drug Discovery (2008) 7(9):771-82. doi: $10.1038 / \mathrm{nrd} 2614$

62. Shen Z, Liu T, Li Y, Lau J, Yang Z, Fan W, et al. Fenton-ReactionAcceleratable Magnetic Nanoparticles for Ferroptosis Therapy of Orthotopic Brain Tumors. ACS Nano (2018) 12(11):11355-65. doi: 10.1021/ acsnano.8b06201

63. Jiang Q, Wang K, Zhang X, Ouyang B, Liu H, Pang Z, et al. Platelet Membrane-Camouflaged Magnetic Nanoparticles for Ferroptosis-Enhanced Cancer Immunotherapy. Small (2020) 16(22):e2001704. doi: 10.1002/ smll.202001704

64. Kim SE, Zhang L, Ma K, Riegman M, Chen F, Ingold I, et al. Ultrasmall Nanoparticles Induce Ferroptosis in Nutrient-Deprived Cancer Cells and Suppress Tumour Growth. Nat Nanotechnol (2016) 11(11):977-85. doi: 10.1038/nnano.2016.164

65. Li L, Sun S, Tan L, Wang Y, Wang L, Zhang Z, et al. Polystyrene Nanoparticles Reduced ROS and Inhibited Ferroptosis by Triggering Lysosome Stress and TFEB Nucleus Translocation in a Size-Dependent Manner. Nano Lett (2019) 19(11):7781-92. doi: 10.1021/acs.nanolett.9b02795

66. Li L, Sun S, Tan L, Wang Y, Wang L, Zhang Z, et al. Correction to "Polystyrene Nanoparticles Reduced ROS and Inhibited Ferroptosis by
Triggering Lysosome Stress and TFEB Nucleus Translocation in a SizeDependent Manner". Nano Lett (2019) 19(12):9170. doi: 10.1021/ acs.nanolett.9b04770

67. Zhang J, Yang J, Zuo T, Ma S, Xokrat N, Hu Z, et al. Heparanase-Driven Sequential Released Nanoparticles for Ferroptosis and Tumor Microenvironment Modulations Synergism in Breast Cancer Therapy. Biomaterials (2021) 266:120429. doi: 10.1016/j.biomaterials.2020.120429

68. Feng H, Schorpp K, Jin J, Yozwiak CE, Hoffstrom BG, Decker AM, et al. Transferrin Receptor Is a Specific Ferroptosis Marker. Cell Rep (2020) 30 (10):3411-23.e7. doi: 10.1016/j.celrep.2020.02.049

69. Chen X, Comish PB, Tang D, Kang R. Characteristics and Biomarkers of Ferroptosis. Front Cell Dev Biol (2021) 9:637162. doi: 10.3389/fcell.2021.637162

70. Lee YS, Lee DH, Choudry HA, Bartlett DL, Lee YJ. Ferroptosis-Induced Endoplasmic Reticulum Stress: Cross-Talk Between Ferroptosis and Apoptosis. Mol Cancer Res (2018) 16(7):1073-6. doi: 10.1158/15417786.Mcr-18-0055

71. Zhou B, Liu J, Kang R, Klionsky DJ, Kroemer G, Tang D. Ferroptosis Is a Type of Autophagy-Dependent Cell Death. Semin Cancer Biol (2020) 66:89-100. doi: 10.1016/j.semcancer.2019.03.002

72. Stockwell BR, Jiang X, Gu W. Emerging Mechanisms and Disease Relevance of Ferroptosis. Trends Cell Biol (2020) 30(6):478-90. doi: 10.1016/ j.tcb.2020.02.009

73. Chen C, Dubin R, Kim MC. Orphan Drugs and Rare Diseases: A Scientometric Review (2000-2014). Expert Opin Orphan Drugs (2014) 2 (7):709-24. doi: 10.1517/21678707.2014.920251

74. Eagle H. Nutrition Needs of Mammalian Cells in Tissue Culture. Science (1955) 122(3168):501-14. doi: 10.1126/science.122.3168.501

75. Bannai S, Tsukeda H, Okumura H. Effect of Antioxidants on Cultured Human Diploid Fibroblasts Exposed to Cystine-Free Medium. Biochem Biophys Res Commun (1977) 74(4):1582-8. doi: 10.1016/0006-291x(77)90623-4

76. Murphy TH, Miyamoto M, Sastre A, Schnaar RL, Coyle JT. Glutamate Toxicity in a Neuronal Cell Line Involves Inhibition of Cystine Transport Leading to Oxidative Stress. Neuron (1989) 2(6):1547-58. doi: 10.1016/08966273(89)90043-3

77. Dixon SJ, Patel DN, Welsch M, Skouta R, Lee ED, Hayano M, et al. Pharmacological Inhibition of Cystine-Glutamate Exchange Induces Endoplasmic Reticulum Stress and Ferroptosis. Elife (2014) 3:e02523. doi: $10.7554 /$ eLife. 02523

78. Angeli JPF, Shah R, Pratt DA, Conrad M. Ferroptosis Inhibition: Mechanisms and Opportunities. Trends Pharmacol Sci (2017) 38(5):489-98. doi: 10.1016/ j.tips.2017.02.005

79. Yu H, Guo P, Xie X, Wang Y, Chen G. Ferroptosis, a New Form of Cell Death, and its Relationships With Tumourous Diseases. J Cell Mol Med (2017) 21 (4):648-57. doi: 10.1111/jcmm.13008

80. Kwon MY, Park E, Lee SJ, Chung SW. Heme Oxygenase-1 Accelerates Erastin-Induced Ferroptotic Cell Death. Oncotarget (2015) 6(27):24393-403. doi: 10.18632 /oncotarget.5162

81. Mancias JD, Wang X, Gygi SP, Harper JW, Kimmelman AC. Quantitative Proteomics Identifies NCOA4 as the Cargo Receptor Mediating Ferritinophagy. Nature (2014) 509(7498):105-9. doi: 10.1038/nature13148

82. Zhang Z, Yao Z, Wang L, Ding H, Shao J, Chen A, et al. Activation of Ferritinophagy Is Required for the RNA-Binding Protein ELAVL1/HuR to Regulate Ferroptosis in Hepatic Stellate Cells. Autophagy (2018) 14(12):2083103. doi: $10.1080 / 15548627.2018 .1503146$

83. Torii S, Shintoku R, Kubota C, Yaegashi M, Torii R, Sasaki M, et al. An Essential Role for Functional Lysosomes in Ferroptosis of Cancer Cells. Biochem J (2016) 473(6):769-77. doi: 10.1042/bj20150658

84. Wang SJ, Li D, Ou Y, Jiang L, Chen Y, Zhao Y, et al. Acetylation Is Crucial for P53-Mediated Ferroptosis and Tumor Suppression. Cell Rep (2016) 17 (2):366-73. doi: 10.1016/j.celrep.2016.09.022

85. Devos D, Moreau C, Kyheng M, Garcon G, Rolland AS, Blasco H, et al. A Ferroptosis-Based Panel of Prognostic Biomarkers for Amyotrophic Lateral Sclerosis. Sci Rep (2019) 9(1):2918. doi: 10.1038/s41598-019-39739-5

86. Yan WT, Lu S, Yang YD, Ning WY, Cai Y, Hu XM, et al. Research Trends, Hot Spots and Prospects for Necroptosis in the Field of Neuroscience. Neural Regener Res (2021) 16(8):1628-37. doi: 10.4103/1673-5374.303032

87. Qiu Y, Cao Y, Cao W, Jia Y, Lu N. The Application of Ferroptosis in Diseases. Pharmacol Res (2020) 159:104919. doi: 10.1016/j.phrs.2020.104919 
88. Stockwell BR, Jiang X. The Chemistry and Biology of Ferroptosis. Cell Chem Biol (2020) 27(4):365-75. doi: 10.1016/j.chembiol.2020.03.013

Conflict of Interest: The authors declare that the research was conducted in the absence of any commercial or financial relationships that could be construed as a potential conflict of interest.
Copyright $\odot 2021$ Zhang, Song, Xu, Fan, Wang, Tian, Ju and Xu. This is an openaccess article distributed under the terms of the Creative Commons Attribution License (CC BY). The use, distribution or reproduction in other forums is permitted, provided the original author(s) and the copyright owner(s) are credited and that the original publication in this journal is cited, in accordance with accepted academic practice. No use, distribution or reproduction is permitted which does not comply with these terms. 